\title{
Caracterización del subsuelo y análisis de riesgos geotécnicos asociados a las arcillas expansivas de la ciudad de Tuxtla Gutiérrez
}

\section{Subsoil Characterization and Analysis of Geotechnical Risks Associated to the Expansive Clays of Tuxtla Gutiérrez City}

\author{
Ordóñez-Ruiz Jorge \\ Instituto de Ingeniería \\ Universidad Nacional Autónoma de México \\ Correo:jorgeor_01@hotmail.com \\ Auvinet-Guichard Gabriel \\ Instituto de Ingeniería \\ Universidad Nacional Autónoma de México \\ Correo:gauvinetg@iingen.unam.mx
}

\author{
Juárez-Camarena Moisés \\ Instituto de Ingeniería \\ Universidad Nacional Autónoma de México \\ Correo:mjuarezc@iingen.unam.mx
}

Información del artículo: recibido: abril de 2014, reevaluado: mayo 2014, aceptado: agosto de 2014

\section{Resumen}

Se presenta una investigación realizada para la caracterización geológica y geotécnica del subsuelo y la evaluación de riesgos geotécnicos en la ciudad de Tuxtla Gutiérrez, Chiapas. Se presentan los resultados obtenidos para la llamada zona geotécnica " $\mathrm{A}$ ", donde se realizaron sondeos y ensayes experimentales para determinar las propiedades índice y mecánicas de los estratos de arcillas de características expansivas, que conforman esta zona urbana. Se aplican métodos indirectos y directos, para identificar, clasificar y determinar el potencial y la presión de expansión de las arcillas. Se aplica la geoestadística como herramienta matemática para determinar la variación espacial del contenido de agua y del potencial de expansión de las arcillas. Se proponen dos métodos: el primero para identificar y clasificar el potencial de expansión de las arcillas y el segundo para identificar y clasificar los tipos de riesgos. Se construyen mapas de riesgos geotécnicos. Para mitigar los riesgos, se propone recurrir a un geomaterial estabilizado, producto de la mezcla de arcilla expansiva y granzón (agregados calcáreos), en proporción 1:1, que disminuye la expansión unitaria de $25 \%$ a $75 \%$. Los resultados de esta investigación contribuirán a resolver la problemática de las fallas estructurales y pérdidas sustanciales comunes en las obras de ingeniería civil de la ciudad que han sido construidas en las últimas cuatro décadas.

\section{Descriptores:}

- caracterización

- riesgos geotécnicos

- arcillas expansivas

- variación espacial

- granzón 


\begin{abstract}
An investigation was performed for subsurface characterization and analysis of geotechnical risks in the City of Tuxtla Gutierrez. Surveys and tests were done to determine the rate and mechanical properties of expansive clays strata found in geotechnical area "A". Direct and indirect geotechnical techniques were used to identify and classify the clays and to determine their expansion potential. Geostatistical tools were used for determining the spatial variation of the water content and expansion parameters of clays. Two methods were proposed, first to identify and classify the potential expansion of the clays and second to identify and classify the types of geotechnical risks. Geotechnical risk maps were constructed. Stabilized product of the mixture of swelling clay and granzón, geomaterial 1:1, which reduces the unit expansion of $25 \%$ to $75 \%$ is proposed. The results of this research will contribute to solving the problem of structural failures and loss of multimillion civil engineering works that have been registered in the city in the last four decades.
\end{abstract}

\section{Introducción}

Los daños estructurales causados por las arcillas expansivas a las obras de ingeniería, cimentaciones o pavimentos, causan pérdidas económicas sustanciales y un inadecuado servicio de las construcciones. Este problema ha sido y es motivo de investigación experimental enfocada a la caracterización y mitigación de riesgos geotécnicos.

Los factores que influyen en la expansión pueden dividirse en dos grupos. El primero incluye las condiciones estratigráficas y las propiedades intrínsecas del suelo: tipo de mineral arcilloso, tamaño y superficie específica de partículas, contenido de arcilla y contenido de agua. El segundo grupo abarca las condiciones ambientales: precipitación, evaporación y temperatura.

En la naturaleza, por las condiciones ambientales las arcillas expansivas sufren cambios importantes de contenido de agua: en época de lluvias el estrato superior se satura y en época de estiaje se produce el fenómeno de desecación y esta capa superficial se comporta como un suelo parcialmente saturado. El sistema hidrológico de la cuenca y subcuencas de la ciudad, influye en el comportamiento del subsuelo porque en época de lluvias se presentan corrientes o flujos de agua.

Con el objetivo de ampliar el conocimiento de las características del subsuelo de la ciudad y en particular de las arcillas expansivas de la zona geotécnica " $\mathrm{A}$ ", se elaboró un nuevo programa de investigación experimental de campo y laboratorio.

Se seleccionaron dos sitios donde se han observado daños estructurales muy severos a las obras de ingeniería y que se consideran representativos de las características geotécnicas de la zona geotécnica " $\mathrm{A}$ ".
Para ambos sitios se realizaron sondeos en época de estiaje y lluvias y se extrajeron muestras alteradas e inalteradas para ensayarlas en el laboratorio.

Se obtuvieron las propiedades índice de las arcillas y se determinó su potencial y grado de expansión. Se realizaron ensayes de expansión en el consolidómetro para los sitios seleccionados, en tres modalidades: ensayo consolidación-expansión, ensayo múltiple en el consolidómetro para diferentes contenidos de agua y ensayo doble en el consolidómetro.

Por otra parte, se realizó un análisis geoestadístico para conocer la variación y el comportamiento espacial del contenido de agua y del potencial de expansión. Se construyeron mapas de contorno de estos parámetros. Además, se propusieron criterios para identificar y clasificar el tipo y nivel de peligro y se construyeron mapas de riesgos geotécnicos.

Se presentan brevemente alternativas para la mitigación de riesgos geotécnicos. Se propone recurrir a un geomaterial estabilizado, producto de la mezcla de arcilla expansiva con granzón (geomaterial producto de la roca caliza empleada para la fabricación de agregados) en proporción 1:1. Este material se emplea en sustitución de la arcilla expansiva en la construcción de cimentaciones superficiales, pavimentos rígidos y flexibles.

\section{Descripción de la zona en estudio}

La ciudad de Tuxtla Gutiérrez, capital del estado de Chiapas, se localiza en la región central de la entidad, con las coordenadas siguientes: $16^{\circ} 45^{\prime} 56^{\prime \prime}$ latitud norte y $93^{\circ} 06^{\prime} 56^{\prime \prime}$ longitud oeste, a una altura de $550 \mathrm{msnm}$. Tiene una extensión territorial de $412.4 \mathrm{~km}^{2}$, que representa $0.51 \%$ de la extensión estatal. El tipo de clima es cálido subhúmedo; la temperatura media anual es de 
$24.5^{\circ} \mathrm{C}$. La temporada de lluvias se presenta entre los meses de mayo y octubre y la de estiaje de noviembre a abril. La precipitación anual es alrededor de $900 \mathrm{~mm}$.

\section{Topografía}

En la figura 1, se presenta el plano topográfico de la ciudad. La orografía de Tuxtla Gutiérrez está conformada por dos montañas separadas por una planicie, la primera montaña se eleva a partir del libramiento norte y alcanza la cota del margen superior del Cañón del Sumidero, de aproximadamente $800 \mathrm{msnm}$. La planicie del centro de la ciudad registra cotas de $505 \mathrm{msnm}$; el cauce del río Sabinal desciende hasta los 500 msnm y constituye el dren pluvial más importante de la ciudad. La cota de referencia (526 msnm) está materializada en el asta bandera del Zócalo. En la parte sur de la ciudad, la segunda montaña inicia a partir del libramiento sur y se extiende hasta la meseta de Copoya que alcanza niveles topográficos de 700 msnm.

\section{Contribución geológica}

El valle de Tuxtla Gutiérrez está enmarcado geológicamente por secuencias de sedimentos marinos de edad correspondiente al Cretácico inferior-terciario medio, deformadas por dos eventos orogénicos, uno del Cretácico superior-Terciario inferior (orogenia Laramide) y otro del Terciario medio (orogenia Chiapaneca). La figura 2 describe el entorno geológico estructural del valle (Zúñiga y Ordóñez, 2013).

La primera de estas orogenias deformó la secuencia hasta las unidades del Cretácico superior y originó los pliegues y cabalgaduras más antiguos que se observan en las cercanías de Tuxtla, los cuales, al igual que las formaciones del resto

Figura 2. Mapa del entorno geológico estructural del valle de Tuxtla Gutiérrez (Zúñiga y Ordóñez, 2013)

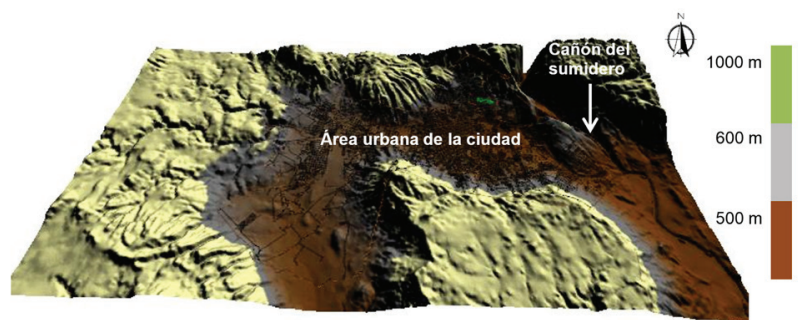

Figura 1. Plano topográfico de la ciudad de Tuxtla Gutiérrez. (Fuente: INEGI, 2009)

\section{TUXTLA GUTIÉRREZ}

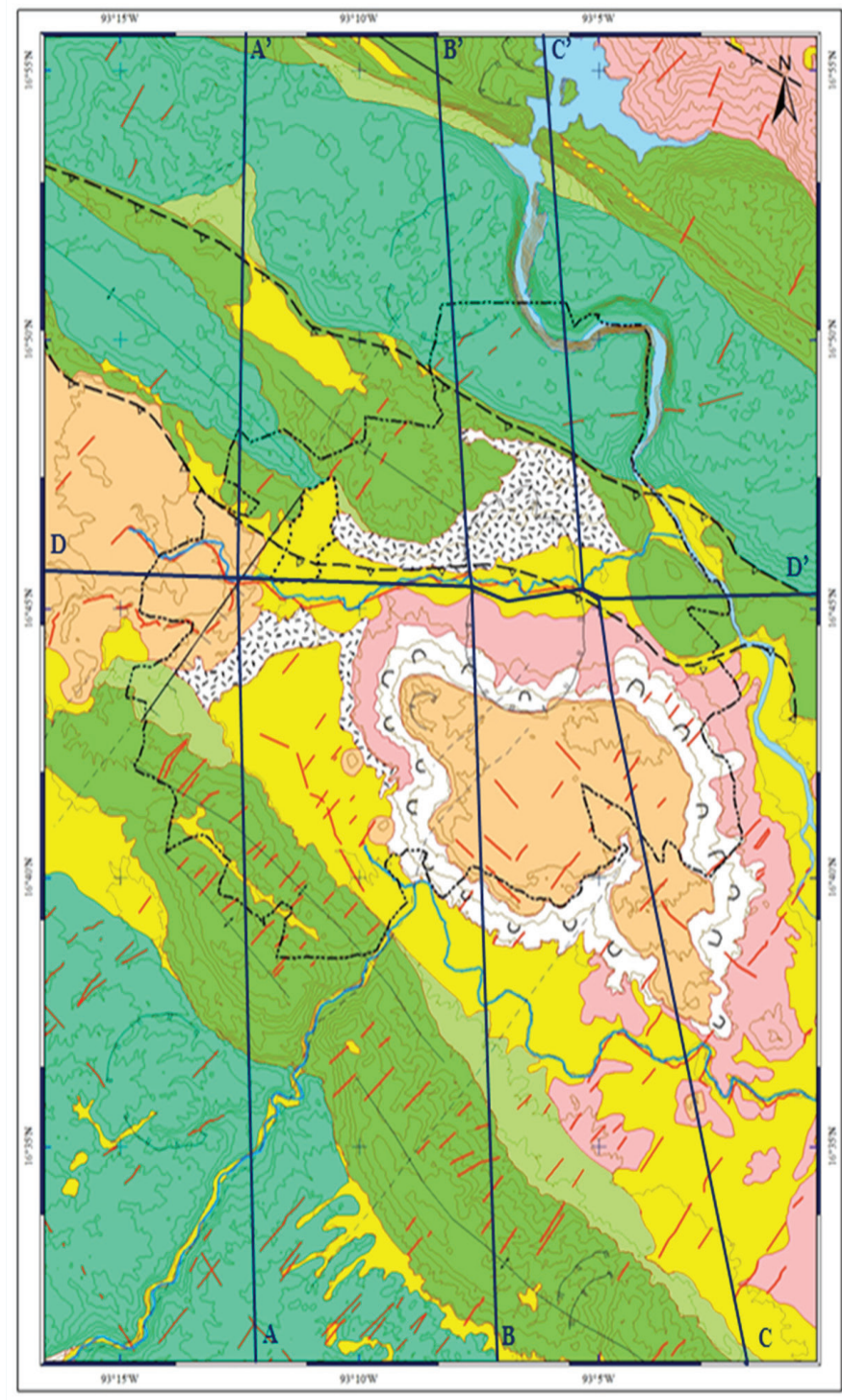

CARTA GEOLÓGICA

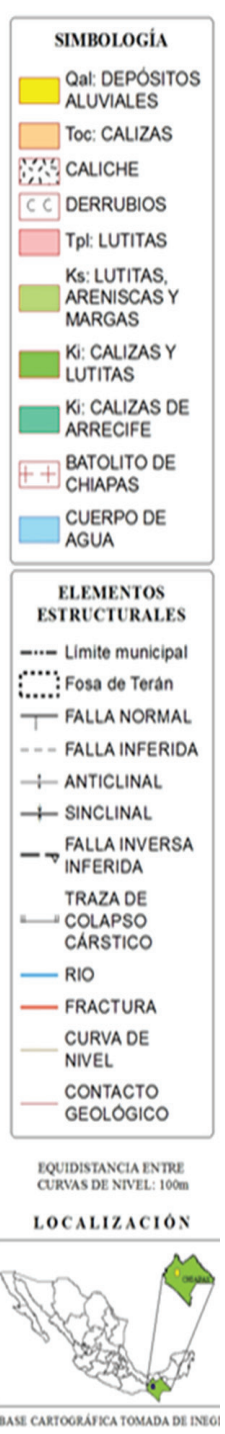

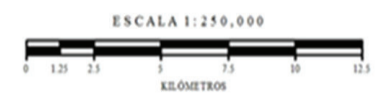




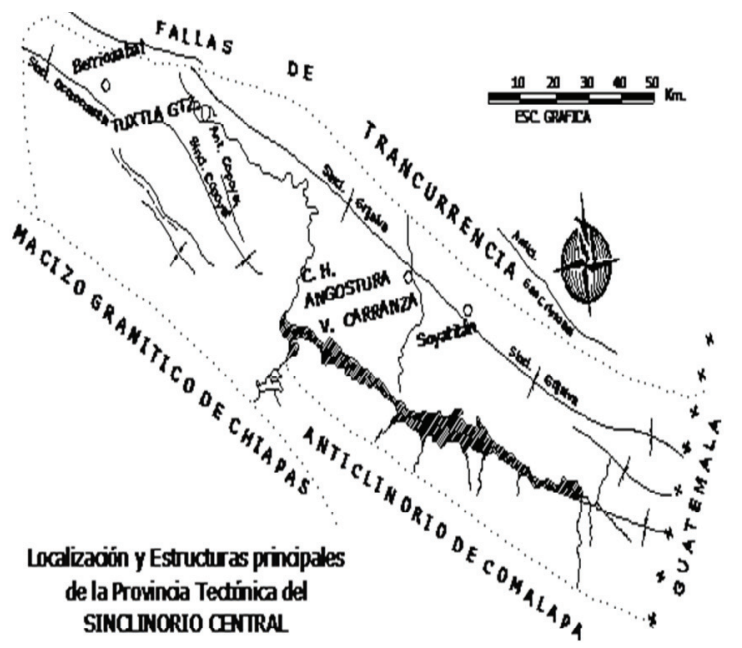

Figura 3. Sinclinorio Central que abarca al valle de la Ciudad de Tuxtla Gutiérrez (De la Rosa et al., 1989)

de la Sierra Madre Oriental, presentan orientaciones NW-SE.

En el Terciario medio (Mioceno) se verificó un nuevo impulso tectónico que volvió a deformar la secuencia ya afectada por la orogenia Laramide, comprimiendo aún más los pliegues previos y deformando las secuencias marinas del Terciario medio que se habían formado después de la orogenia Laramide.

El resultado es un paisaje con pliegues intensamente apretados que a manera de isoclinal chocaron desde el norte contra el macizo granítico de Chiapas; en este conjunto litológico deformado destacan amplios pliegues formados por secuencias calcáreas de arrecife que sufrieron los empujes orogénicos, deformándose con respecto a las secuencias de estratos más delgados que chocaron contra ellos. Al pie de los pliegues se encuentran las secuencias aluvio-fluviales y hasta derrubios asociados a la erosión e inestabilidad de las formaciones marinas plegadas.

En la región sur de la ciudad dominan los flancos septentrionales de la meseta Copoya, al norte de la ciudad se distinguen cubiertas calichosas irregulares que descansan sobre las calizas formando suelos resistentes; hacia el sur se encuentran las secuencias aluviales modernas del río Sabinal que describen una delgada franja orientada casi al W-E, compuesta por capas superficiales de arenas y limos, que descansan sobre capas de espesor irregular formadas por arcillas derivadas de la alteración por intemperismo de las lutitas subyacentes. Conformados en su porción inferior por "lutitas" de edad paleocénica cubiertas por suelos residuales arcillosos derivados del intemperismo de las mismas lutitas; en su porción media-superior dominan derrubios asociados a deslizamientos originados en las por- ciones abruptas más altas de la meseta; estos derrubios están ligados a la estabilidad dictada por el borde sur de un colapso cárstico localizado en la región central de la ciudad.

Hacia la porción occidental del municipio, destaca una franja aluvial de forma rectangular orientada al SW-NE, que se asocia a la fosa tectónica con orientación idéntica denominada como Fosa Terán; esta fosa tectónica debió cortar la meseta Copoya con la zona donde se encuentra el municipio de Berriozábal y debe marcar la existencia de mayores espesores de rellenos aluvio-fluviales.

La situación física del municipio de Tuxtla Gutiérrez se define por la presencia de rasgos geológico-estructurales, que influyen en el comportamiento del subsuelo ante el efecto de las ondas sísmicas y están parcialmente influenciadas por la disposición de las unidades litoestratigráficas. Los rasgos geoestructurales son: la cabalgadura entre dos pliegues o elementos orogénicos que debe pasar aproximadamente por debajo de la traza del río Sabinal. Al norte, se observa un pliegue formado por calizas y calizas intercaladas con "lutitas", todas del Cretácico inferior. Al sur se observan los elementos de un pliegue formado por "lutitas" cubiertas discordantemente por calizas y calizas con intercalaciones de areniscas del Terciario medio formando la meseta de Copoya. El colapso Cárstico localizado en la porción central de la ciudad afecta parcialmente al pliegue del norte y a la meseta de Copoya. Al sur, dentro de este colapso de traza casi elíptica, deben encontrarse rellenos constituidos por rocas fragmentadas asociadas al mismo colapso y también por rellenos aluviales. El río Sabinal presenta un curso de traza quebrada zigzagueante que se liga con un fracturamiento superficial del terreno y drena esta porción de la depresión central.

\section{Sismicidad}

La zona de Tuxtla Gutiérrez se encuentra en el flanco meridional del Sinclinal Central de rumbo NW-SE. Los rasgos estructurales de la región, que aparecen indicados en el plano geológico están representados por fallas y sinclinales de dirección $\mathrm{N} 45^{\circ} \mathrm{W}$, que afectan sobre todo las rocas calcáreas del Cretácico. El Sinclinorio Central colinda con las provincias tectónicas del macizo granítico de Chiapas, las fallas de transcurrencia y el anticlinorio de Comalapa, como se observa en la figura 3.

\section{Hidrología}

El valle de Tuxtla Gutiérrez se enmarca en la cuenca del río Sabinal (Domínguez et al., 1997). El río Sabinal es un 
afluente del río Grijalva y drena una superficie aproximada de $407 \mathrm{~km}^{2}$, del valle de Tuxtla Gutiérrez (López, 2006). Su confluencia con el río Grijalva ocurre a la altura de la meseta conocida como Nido de Águilas, punto de entrada del río al Cañón del Sumidero. La cuenca del río Sabinal está ubicada dentro de la región hidrológica Núm. 30, denominada Grijalva-Usumacinta y en la cuenca administrativa Grijalva-Tuxtla Gutiérrez; está formada por 15 subcuencas (figura 4).

\section{Geotecnia}

\section{Antecedentes}

La línea de investigación: El subsuelo de la ciudad de Tuxtla Gutiérrez, ha permitido avanzar en el conocimiento de las características geotécnicas del subsuelo de la ciudad y elaborar un mapa de zonificación geotécnica (Ordóñez, 2008). Se distinguen tres zonas geotécnicas (A, B y C). En la zona geotécnica $A$, se localizan los suelos expansivos y presentan espesores de 0 y hasta $9 \mathrm{~m}$, abarcan la mayor parte de la extensión territorial de la ciudad. En la zona geotécnica B, se localizan las arcillas "lutitas", le subyace estrato de rocas "lutitas". En la zona geotécnica $\mathrm{C}$, se ubican los estratos de roca calizas y el caliche (suelo producto de la intemperización de las rocas calizas).

\section{Principios de la mecánica de suelos no saturados}

La mecánica de suelos tradicional se aplica a suelos saturados o secos. Últimamente, la mecánica de los suelos no saturados está tomando una gran importancia (Fredlund y Rahardjo, 1993). Para suelos saturados, Terzaghi (1936) definió el concepto de esfuerzo efectivo $\left(\sigma^{\prime}\right)$, a partir de

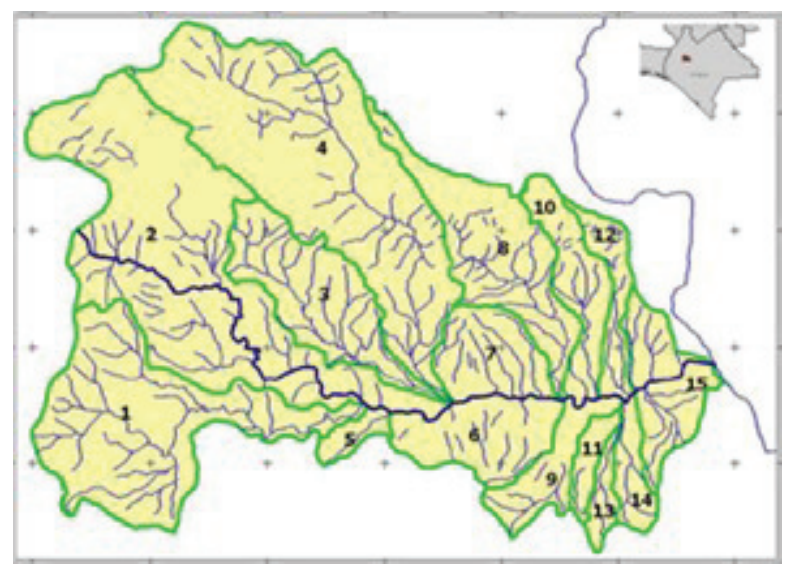

Figura 4. Sistema de drenaje de la cuenca del río Sabinal (Domínguez et al., 1997) la expresión $\sigma^{\prime}=\sigma-u_{w}$. Los procesos de cambios de volumen y las características de resistencia al esfuerzo cortante de un suelo saturado están controlados por los esfuerzos efectivos. En la estructura de los suelos no saturados generalmente se consideran tres fases (Lambe y Whitman, 1979). Recientemente, la capa contráctil, interfaz agua-aire, se introdujo como una cuarta fase independiente para el análisis teórico de esfuerzos en el suelo (Fredlund y Morgenstern, 1977). En la figura 5, se presenta un elemento de suelo parcialmente saturado, con las tres fases: aire, agua, sólidos y la capa contráctil. La fase de aire consiste en oclusión de bulbos de aire, el fluido es significativamente compresible.

En los suelos parcialmente saturados la presión en el agua puede ser negativa (succión) en relación con la presión del aire. El agua está sujeta a fenómenos de capilaridad y de absorción que, al combinarse, generan una matriz de esfuerzos al interior de la estructura del suelo, produciendo la succión matricial. Bishop (1959) propuso la ecuación: $\sigma^{\prime}=\left(\sigma-u_{a}\right)+\chi\left(u_{a}-u_{w}\right)$, para el análisis de esfuerzos efectivos en suelos no saturados, $u_{a}$ es la presión de poro del aire, $u_{w}$ es la presión de poro del agua.

Cuando el valor de $u_{w}$ se aproxima a $u_{a}$ la succión decrece y el grado de saturación se incrementa; cuando $\chi$ tiende a la unidad, entonces el suelo está saturado; el parámetro $\chi$ es unitario para un suelo saturado y nulo para un suelo seco. En época de estiaje, el secado gradual de la masa de suelo, causa agrietamientos.

Las propiedades índice son útiles para identificar los suelos expansivos; las más importantes son: el límite líquido, el contenido de agua y el porcentaje de partículas finas. El límite líquido y el contenido de agua están correlacionados con la mineralogía. El contenido de partículas finas menores a $2 \mu$ (coloides) define la actividad de las arcillas.

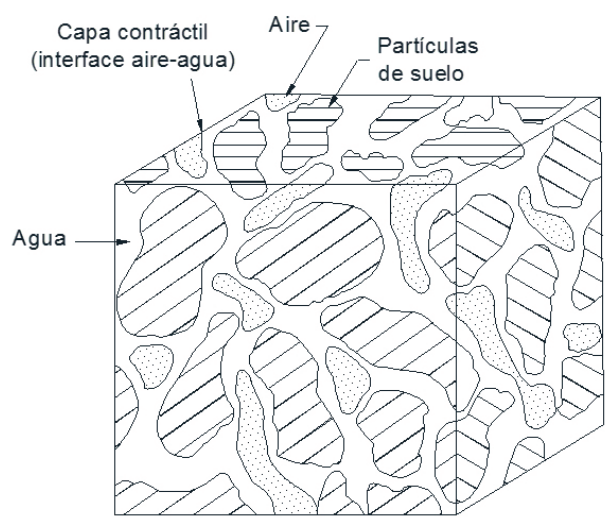

Figura 5. Elemento de suelo parcialmente saturado con una fase de aire continua (Fredlund y Morgenstern, 1977) 
La expansión de las arcillas está asociada con el ciclo hidrológico y climático anual y con las actividades del hombre. La absorción de agua puede tener su origen en infiltraciones provocadas por: precipitaciones pluviales, flujos o corrientes de agua en el suelo, variación del nivel de aguas freáticas, riego y fugas en ductos de agua potable o de drenaje.

\section{Investigación experimental}

Para esta investigación se seleccionaron dos sitios de la zona geotécnica A. El sitio 1 (Parque Caña Hueca) corresponde a la subzona poniente y el sitio 2 (Col. Las Palmas, Electricistas y el Retiro), a la subzona oriente. Se diseñó un programa de sondeos para caracterizar las áreas de los sitios con mayor registro de daños a las obras de ingeniería. Se definieron mallas de ubicación de sondeos para las épocas de estiaje y de lluvias.

Se realizaron sondeos tipo pozos a cielo abierto (PCA), sondeos profundos mixtos (SPM) combinando la prueba de penetración estándar (SPT), con la extracción de muestras alteradas e inalteradas. Con las muestras alteradas se realizaron ensayes para determinar las propiedades índice de los suelos: granulometría, incluyendo la prueba del hidrómetro, límites de consistencia, contracción lineal, densidad específica de las partículas sólidas y pesos volumétricos. Con las muestras inalteradas se determinaron las propiedades mecánicas de las arcillas; se realizaron ensayes en el consolidómetro en las tres modalidades: consolidación-expansión, consolidación para diferentes contenidos de agua y doble ensayo en el consolidómetro (ASTM, 1971). En el consolidómetro, se ensayaron muestras con contenido de agua inicial correspondiente tanto a la época de estiaje como a la época de lluvias. En las arcillas expansivas el problema geotécnico no es de capacidad de carga, sin embargo, se realizaron en la misma forma ensayos de compresión simple y triaxiales no consolidadas-no drenadas (UU) y de valor relativo de soporte $(C B R)$. Los parámetros de resistencia: compresión simple $\left(q_{u}\right)$, cohesión $\left(C_{u u}\right)$, fricción $\left(\phi_{u}\right)$ y el $C B R$ registran valores bajos para la época de lluvias, porque el estrato superficial del suelo de 0.25 a $3 \mathrm{~m}$ de profundidad alcanza porcentajes altos de contenido de agua (Ordóñez, 2013).

Con los datos geotécnicos obtenidos de los sondeos y ensayes experimentales, se realizó la caracterización geotécnica de las arcillas expansivas de la zona A. Se aplicaron los métodos indirectos para la definición del potencial de expansión de las arcillas y con los métodos directos, se determinó la presión de expansión para diferentes contenidos de agua iniciales en los especímenes ensayados.
Se aplicó la geoestadística como herramienta matemática para determinar la variación espacial de los parámetros geotécnicos analizados con este método. Se construyeron mapas de contornos, de desviación estándar de estimación y de riesgos geotécnicos.

Para la definición de medidas de mitigación de riesgos geotécnicos, se realizaron ensayes en el laboratorio con mezclas de arcillas expansivas, arenas, caliche y granzón.

\section{Métodos indirectos}

Los métodos o criterios empleados para definir las características de expansividad de las arcillas de la ciudad se describen a continuación. Para la aplicación de estos métodos, se emplearon los resultados experimentales de la tabla del Anexo 1.

\section{Criterio de Dakshanamurthy y Raman (1973)}

Dakshanamurthy y Raman (1973), basados en la carta de plasticidad de Casagrande (1948) modificada por Dumbleton (1968), propusieron definir el grado de expansividad de las arcillas en función del límite líquido $\left(w_{L}, \%\right)$, límite de contracción volumétrica $\left(w_{S}, \%\right)$, índice de plasticidad (PI, \%) e índice de contracción $\left(I_{S}, \%\right)$, $I_{S}=w_{L}-w_{S}$. Los pares de valores $\left(w_{L^{\prime}} P I\right)$, proporcionan una evaluación de la plasticidad del suelo y el par $\left(w_{L}, I_{S}\right)$, proporciona el grado de expansividad de las arcillas, como se indica en la figura 6 . De acuerdo con estos autores, la expansión vertical unitaria ( $E$, \% expansión vertical unitaria) de un estrato de arcilla puede calcularse con la siguiente expresión

$E(\%)=\left(41.13 * 10^{-5}\right)\left(I_{S}^{2.67}\right)$

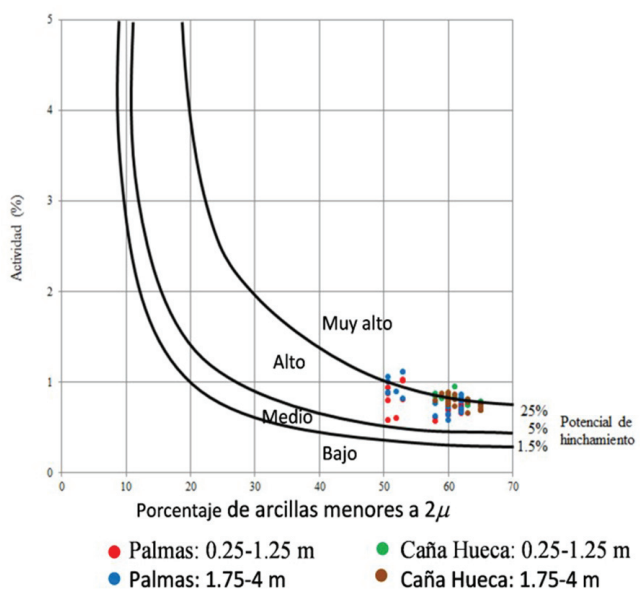

Figura 6. Clasificación de la expansividad en función del límite líquido y del índice de contracción (Dakshanamurthy y Raman, 1973) 
Criterio de Seed, Woodward y Lungren (1962)

Este método (Seed et al., 1962) se fundamenta en la "actividad" de la arcilla. En su trabajo, los autores indican la necesidad de modificar el concepto original de actividad, establecido por Skempton (1953), a partir de la inclinación de la línea que relaciona el índice de plasticidad $(P I)$ y el porcentaje $(C)$ de partículas arcillosas menores a $2 \mu$. Para suelos naturales, la expresión para calcular la actividad de la arcilla es la siguiente

$\mathrm{A}=\frac{(P I)}{(C-5)}$

donde

$A=$ actividad de la arcilla

$P I=$ índice de plasticidad

$C=$ contenido de arcilla

Seed et al. (1962), realizaron ensayos en el consolidómetro con muestras de arcillas compactadas bajo una carga de $6.89 \mathrm{kPa}$ con el peso volumétrico seco máximo y el contenido de agua óptimo (Próctor estándar). Con los resultados de la expansión libre, establecieron correlaciones estadísticas con el contenido de arcilla y la actividad y propusieron calcular el potencial de expansión $(E)$, en por ciento, con la siguiente expresión

$E(\%)=(3.6)\left(10^{-5}\right)\left(A^{2.44}\right)\left(C^{3.44}\right)$

Los autores proponen clasificar el potencial de expansión como se indica en la figura 7 .

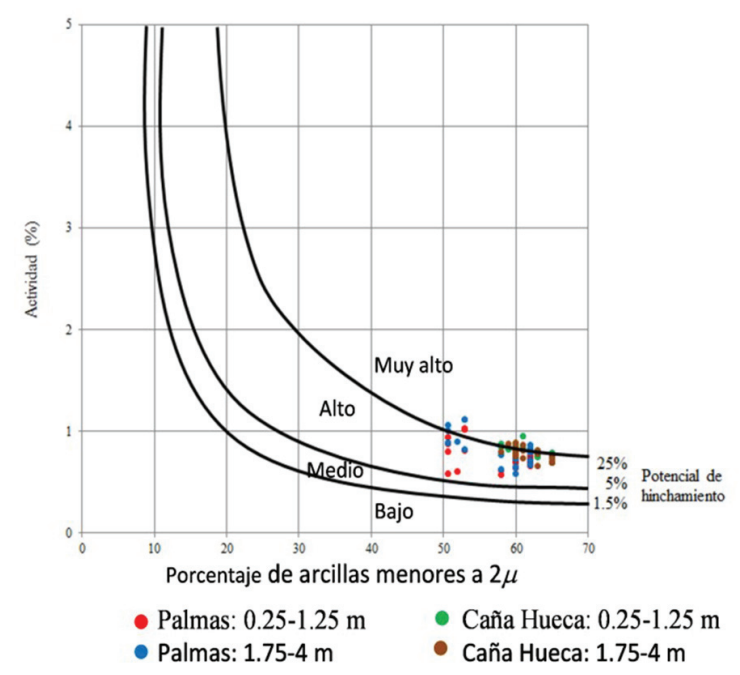

Figura 7. Clasificación del potencial de expansión (Seed et al., 962)
Criterio de Merwe (1964 y 1975)

Entre los criterios propuestos para clasificar el potencial de expansión de las arcillas, a partir de la actividad de las partículas sólidas, el $P I$ y el $\%$ de partículas $\leq 2 \mu$, debe destacarse el propuesto inicialmente por Merwe (1964) y modificado por el mismo autor en 1975. La figura 8 permite determinar el potencial de expansión de las arcillas: bajo, medio, alto y muy alto. En esta misma figura se muestran los resultados obtenidos con los datos de los sitios 1 y 2 (Anexo 1).

Con los datos de las propiedades índice del Anexo 1 , los tres métodos expuestos coinciden en que las arcillas de la ciudad presentan características de media, alta a muy alta expansividad.

\section{Método de Weston (1980)}

Weston (1980) realizó estudios para definir la correlación entre la expansión y otros parámetros que influyen en el fenómeno de expansión de las arcillas. Desarrolló un amplio estudio estadístico de regresión multilineal. Realizó ensayos en el consolidómetro con inundación; las muestras inalteradas se ensayaron con diferentes contenidos de agua inicial y con cargas variables.

Del modelo experimental desarrollado para determinar la expansión de los suelos, se obtuvo la expresión siguiente

$E(\%)=(0.000411)\left(w_{L w}\right)^{4.17}(p)^{-0.386}\left(w_{i}\right)^{-2.33}$

donde $w_{L w}(\%)$, es el límite ponderado y se determina con el producto $\left(w_{L}\right)(\%<$ malla \#40), $p$ es la carga o pre-

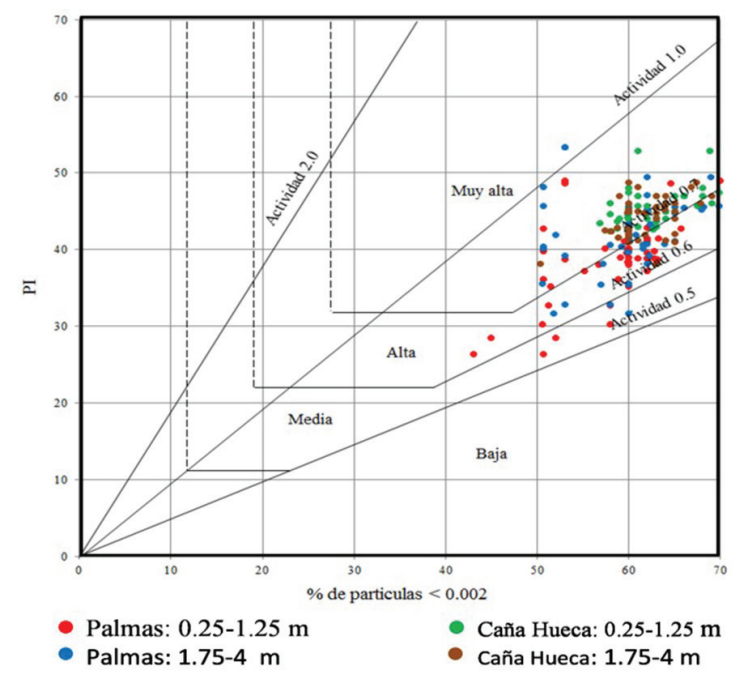

Figura 8. Gráfico de expansividad modificado por Merwe $(1964,1975)$ 
sión vertical $(\mathrm{kPa}), w_{i}(\%)$ es el contenido de agua inicial de la muestra de suelo ensayada en el consolidómetro y $E(\%)$ es la expansión unitaria.

Los ensayes realizados en el presente estudio indican que, de los métodos indirectos, este es el que permite estimar con mayor precisión el porcentaje de expansión de las arcillas.

\section{Métodos directos}

La investigación experimental consistió en realizar los siguientes ensayos: El ensayo de consolidación-expansión (edómetro simple), el ensayo múltiple en el consolidómetro (edómetro múltiple) y el doble ensayo en el consolidómetro (doble edómetro). Estos ensayos son relativamente fáciles de realizar y proporcionan información muy confiable respecto al potencial y a la presión de expansión de los estratos de arcillas. Los ensayos deben realizarse siguiendo secuencias de aplicación de cargas y sobrecargas que simulen las condiciones de esfuerzos y deformabilidad en el sitio para las condiciones de un estrato de suelo saturado y no saturado (Fredlund y Rahardjo, 1993). En este artículo se presentan los resultados obtenidos con los materiales del sitio 2, por considerar que es la zona urbana con registros de mayores daños estructurales y pérdidas económicas.

\section{Sitio 2: Col. Las Palmas, Electricistas y el Retiro}

En la tabla 1, se presentan las propiedades índice de las muestras ensayadas en el consolidómetro para las diferentes modalidades.

\section{Ensayo de consolidación-expansión}

Se presentan los resultados de un ensayo de consolidación-expansión realizado con una muestra del sondeo A-040.

Sobre el espécimen de suelo inalterado obtenido a la profundidad $h=2.5 \mathrm{~m}$, se aplicó una presión inicial de $\sigma_{o}=10 \mathrm{kPa}$. El contenido de agua inicial fue $w_{i}=23.86 \%$, y el grado de saturación $\mathrm{Sr}=80 \%$. Se procedió a la saturación del espécimen $\mathrm{y}$, al término del ensayo, el conte-

Tabla 1. Parámetros geotécnicos de las muestras ensayadas en el consolidómetro

\begin{tabular}{cccccccc}
\hline $\begin{array}{c}\text { Clave de } \\
\text { sondeo }\end{array}$ & $\begin{array}{c}\text { Prof. } \\
(\mathrm{m})\end{array}$ & $\begin{array}{c}w_{L} \\
(\%)\end{array}$ & $\begin{array}{c}\gamma_{m} \\
\left(\mathrm{~g} / \mathrm{cm}^{3}\right)\end{array}$ & $\begin{array}{c}\gamma_{d 1} \\
\left(\mathrm{~g} / \mathrm{cm}^{3}\right)\end{array}$ & $\begin{array}{c}\gamma_{d 2} \\
\left(\mathrm{~g} / \mathrm{cm}^{3}\right)\end{array}$ & $\begin{array}{c}\gamma_{d 3} \\
\left(\mathrm{~g} / \mathrm{cm}^{3}\right)\end{array}$ & $\begin{array}{c}\gamma_{d 4} \\
\left(\mathrm{~g} / \mathrm{cm}^{3}\right)\end{array}$ \\
\hline A-040 & 2.5 & 68 & 1870 & 1510 & 1400 & - & - \\
A-043 & 2.5 & 62 & 1860 & 1370 & 1400 & 1460 & 1520 \\
A-043 & 2.5 & 68 & 1890 & 1430 & 1510 & - & - \\
\hline
\end{tabular}

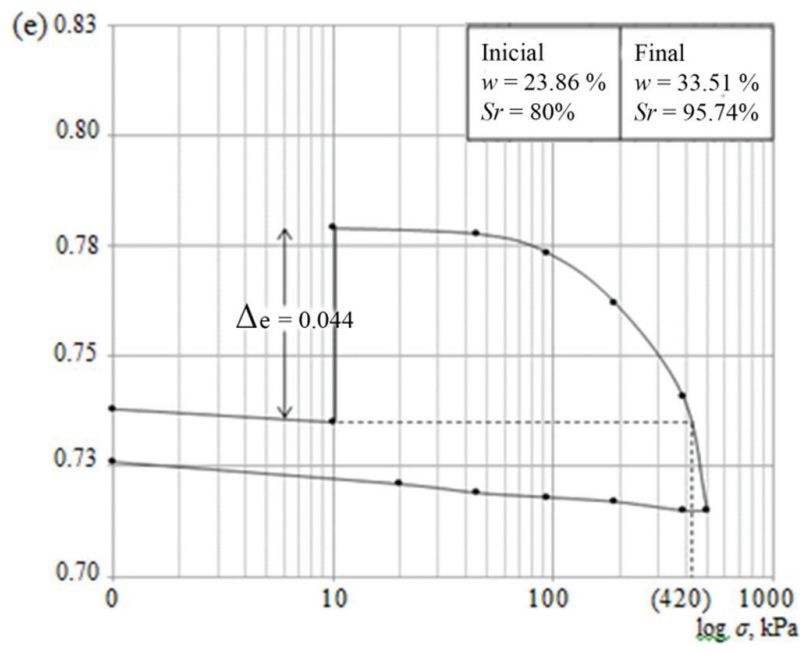

Figura 9. Resultado del ensayo de consolidación-expansión (edómetro simple)

nido de agua registrado fue $w_{f}=33.51 \%$ y el grado de saturación $S r=95.74 \%$. En la figura 9, se presentan los resultados del ensayo. La variación del contenido de agua al final de la prueba fue $\Delta w=9.65 \%$ y la del grado de saturación $\Delta S r=15.74 \%$, con un incremento en la relación de vacíos $\Delta e=0.044$. La expansión unitaria se calcula con la expresión

$$
\Delta \varepsilon_{s}=\frac{e_{2}-e_{1}}{1+e_{1}}
$$

$e_{1}$ y $e_{2}$ son la relación de vacíos de las muestras de arcilla ensayadas. Con los datos del ensayo, $E=2.6 \%$. Para controlar esta expansión $(E=0)$ se requiere una presión $\sigma_{s}=420 \mathrm{kPa}$. Se determinaron los índices de compresión $C_{c}=0.10$ y expansión $C s=0.005$.

Con la expresión de Weston, para $\sigma_{o}=10 \mathrm{kPa} y$ $w_{i}=23.86 \%$ y $w_{f}=33.51 \%, E=(4.56-2.07)=2.5 \%$. Para $\sigma_{s}=420 \mathrm{kPa} \mathrm{y} w_{f}=33.51 \%$, la expansión es $E=0.55 \%$.

Ensayo de consolidación para diferentes contenidos de agua

En la época de estiaje los valores varían de $w=20$ a $22 \%$ y en épocas de lluvias de $w=33$ a $36 \%$. Las muestras corresponden al sondeo con clave A-071. Se ajustaron los contenidos de agua de los especímenes por secado progresivo. Los ensayos se realizaron para los contenidos de agua siguientes: $w=36 \%, w=32.4 \%$ y $w=27.6 \%$ y $w=22.8 \%$. Los resultados se presentan 
en la figura 10 (a,b,c,d y e). Se observa que las curvas a y c no presentan intersección. Por lo contrario, la intersección de las curvas b y d, permite definir una presión de expansión $\sigma_{s}=240 \mathrm{kPa}$, con $\Delta w=9.6 \%$ y $\Delta S r=16.2 \%$. Para las curvas c y d, la presión de expansión correspondiente a la intersección es $\sigma_{s}=55 \mathrm{kPa}$, con $\Delta w=4.8 \%$ y $\Delta S r=10.9 \%$. En la figura 10e se reunieron las cuatro curvas de compresibilidad anteriores.

$\mathrm{Al}$ aplicar la fórmula de Weston, para $\sigma_{s}=55 \mathrm{kPa}$, la expansión es $E=0.66 \%$ y para $\sigma_{s}=240 \mathrm{kPa}$, es $E=0.57 \%$. El método de Weston proporciona resultados con un margen de error $E<1 \%$ sobre la expansión respecto a los valores medidos.

Ensayo doble en el consolidómetro

Para realizar los ensayes dobles en el consolidómetro, se seleccionó el sondeo A-041. El ensaye se realizó con
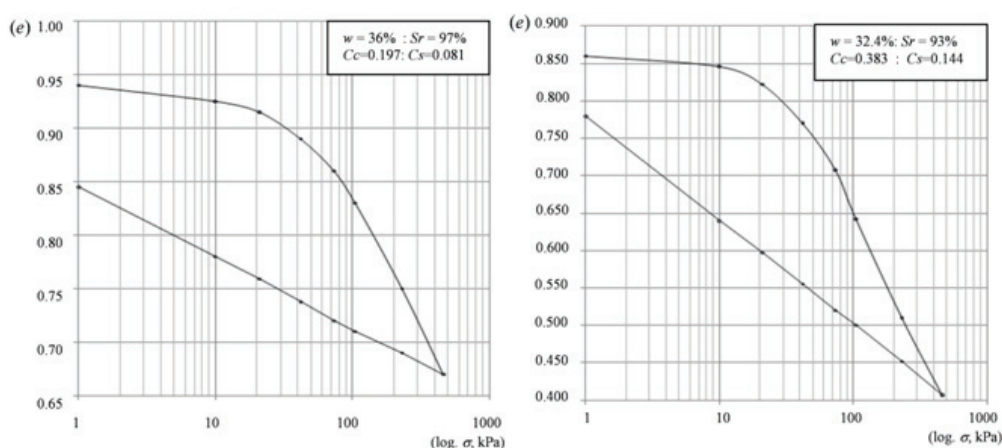

(a)
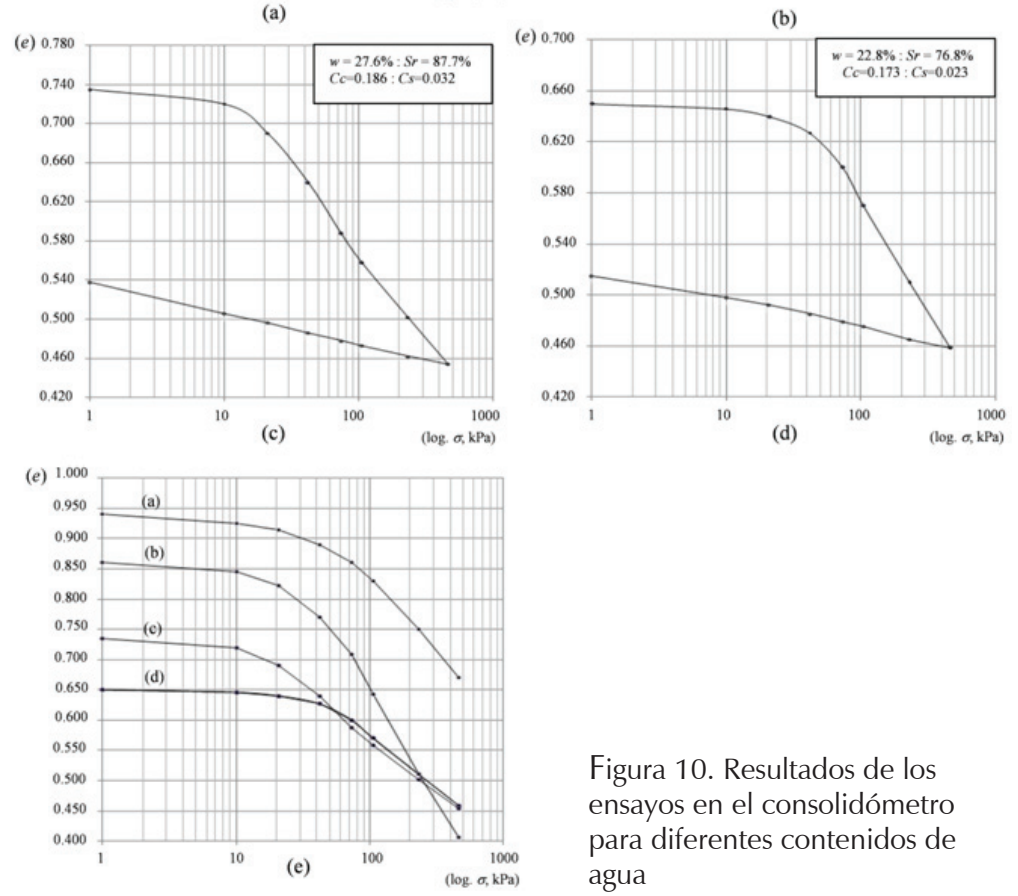

Figura 10. Resultados de los ensayos en el consolidómetro para diferentes contenidos de agua especímenes de la muestra obtenida a la profundidad $h$ $=2.5 \mathrm{~m}$. El espécimen a, se ensayó con $w=32.22 \% \mathrm{y}$ $S r=97.4 \%$ y el espécimen b, con $w=24.91 \%$ y $S r=85.5 \%$, es decir, con diferencias $\Delta w=7.31 \%$ y $\Delta S r=11.90 \%$.

Con estos parámetros geotécnicos se realizaron los ensayes en el consolidómetro, manteniendo constante el contenido de agua de cada espécimen. La presión de expansión registrada fue $\sigma_{s}=350 \mathrm{kPa}$. Los resultados de los dos ensayos se presentan en la figura $11(a, b, c y d)$.

\section{Análisis geoestadístico}

\section{Conceptos generales}

Se considera una variable de interés $V(X)$ definida en los puntos $X$ de un dominio geotécnico estudiado $R^{p}$, $(1,2$ o 3$)$. Esta variable puede ser de tipo físico (contenido de agua del suelo), mecánico (potencial de expansión del suelo) o geométrico (espesor de un estrato de suelo). Si en cada punto del dominio, esta variable se considera aleatoria, el conjunto de estas variables aleatorias constituye un campo aleatorio (Auvinet, 2002).

Para poder describir este campo pueden emplearse los siguientes parámetros y funciones:

\section{Esperanza matemática}

$$
E\{V(X)\}=\mu_{v}(X)
$$

\section{Varianza}

$\sigma_{v}^{2}(X)=\operatorname{Var}[V(X)]$

La raíz cuadrada $\sigma_{v}(X)$ de la varianza se llama desviación estándar y el cociente

$$
C_{v}(X)=\frac{\sigma_{v}(X)}{E\{V(X)\}},
$$

se conoce como coeficiente de variación. La función de autocovarianza,

$$
\begin{aligned}
C_{v}\left(X_{1}, X_{2}\right) & =\operatorname{Cov}\left[V\left(X_{1}\right), V\left(X_{2}\right)\right] \\
& =E\left\{\left[V\left(X_{1}\right)-\mu_{V}\left(X_{1}\right)\right]\left[V\left(X_{2}-\mu_{V}\left(X_{2}\right)\right)\right]\right\}
\end{aligned}
$$

La función de autocovarianza representa el grado de dependencia lineal entre los valores de la propiedad de interés en dos pun- 

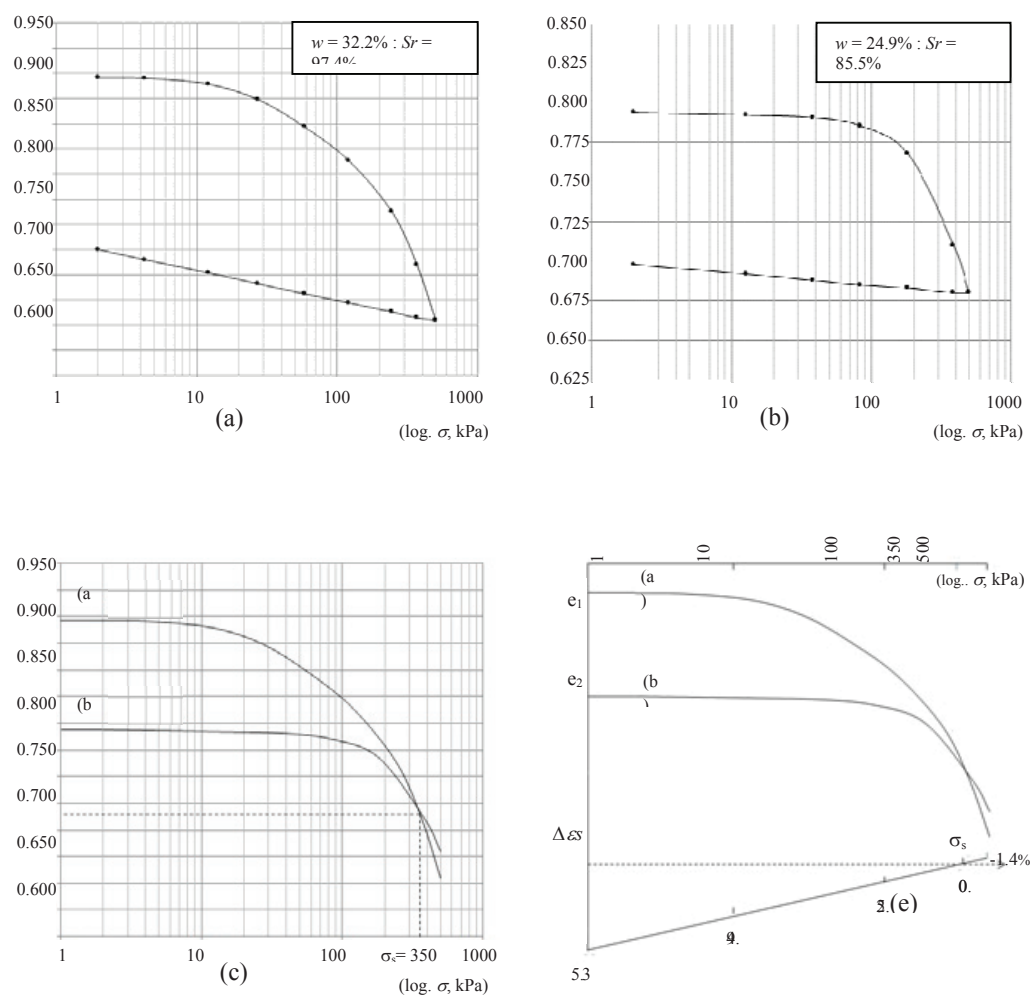

Figura 11. Resultados del doble ensayo en el consolidómetro

tos diferentes del medio. Esta función se puede escribir bajo la forma de un coeficiente de autocorrelación adimensional, cuyo valor queda siempre comprendido entre -1 $\mathrm{y}+1$.

$\rho_{v}\left(X_{1}, X_{2}\right)=\frac{C_{v}\left(X_{1}, X_{2}\right)}{\sigma_{v}\left(X_{1}\right) \sigma_{v}\left(X_{2}\right)}$

La determinación de los parámetros del campo a partir de los datos disponibles recibe generalmente el nombre de análisis estructural. Los parámetros y funciones definidas en las expresiones anteriores se estiman a partir de sondeos continuos, recurriendo a estimadores estadísticos.

El modelado de las variaciones espaciales de las propiedades del suelo con campos aleatorios permite estimar el valor de las propiedades de interés en puntos donde se carezca de medición. Para ello se toma en cuenta la correlación espacial y se recurre usualmente a la técnica del Kriging (Matheron, 1965).

$V^{*}(X)=\sum_{i}^{n} \lambda_{i} V_{i}$
El problema consiste en estimar los parámetros $\lambda_{i}$ que determinen un estimador satisfactorio de $V(X)$. El kriging recurre al "mejor estimador sin sesgo".

Se puede en esta forma interpolar entre los sondeos disponibles de forma racional tomando en cuenta la correlación espacial del campo. Se obtienen así configuraciones virtuales estimadas (cortes, curvas de isovalores) que pueden ser de gran utilidad para el ingeniero. El método proporciona además una desviación estándar de estimación que muestra cómo la precisión de la estimación disminuye con la distancia a los datos originales.

Análisis geoestadístico del sitio 2 (Col. Las Palmas, Electricistas y el Retiro)

Se realizó un estudio de la variación espacial de los parámetros: contenido de agua y potencial de expansión para el sitio 2 de la zona geotécnica $\mathrm{A}$, en la época de estiaje. Esta condición corresponde a la distribución inicial de estos parámetros que puede conducir posteriormente a la expansión de las arcillas. En la figura 12 se presenta el croquis de localización de sondeos.

\section{Parámetros geotécnicos $(w, \%)$ y $\left(E \sigma_{v} \%\right)$.}

Con los datos geotécnicos del contenido de agua y la expansión, se realizó el análisis estructural de los campos. Para el sitio 2, estos campos son prácticamente estacionarios.

En la tabla 2 se presentan los parámetros estadísticos del contenido de agua y en la figura 13 se muestran el histograma y el polígono de frecuencias que presenta una distribución aproximadamente normal.

En la tabla 3 se describen los parámetros estadísticos de la expansión y en la figura 14 se define el histograma y polígono de frecuencias que presenta una distribución aproximadamente normal, pero con asimetría positiva.

En la figura 15, se presenta el mapa de ubicación de sondeos y de cortes virtuales. 


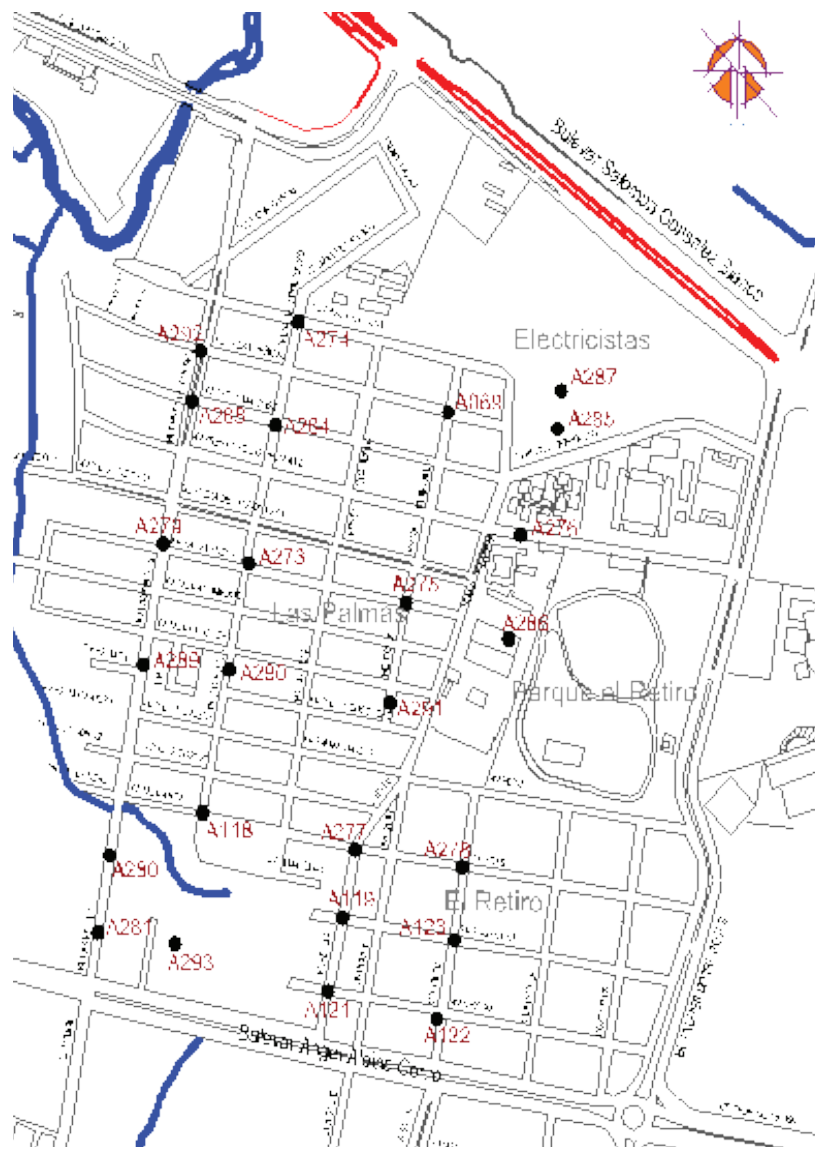

Figura 12. Croquis de localización de los sondeos del sitio 2

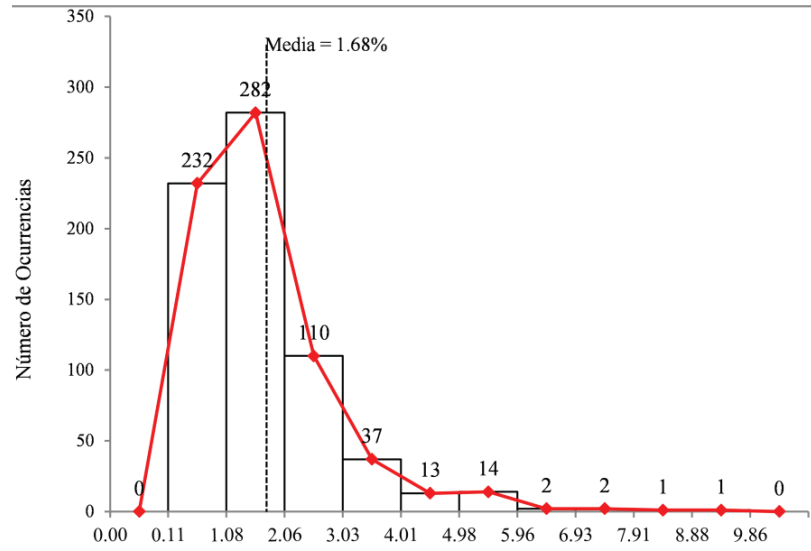

Figura 13. Histograma y polígono de frecuencias para el contenido de agua
Tabla 2. Parámetros estadísticos del contenido de agua

\begin{tabular}{lc}
\hline \multicolumn{1}{c}{ Parámetros estadísticos $(\mathrm{w}, \%)$} \\
\hline Media & 22.63 \\
Mediana & 20 \\
Moda & 23.11 \\
Desviación estándar & 3.6 \\
Varianza & 12.98 \\
Coeficiente de variación & 0.16 \\
Valor mínimo & 14.19 \\
Valor máximo & 39.26 \\
\hline
\end{tabular}

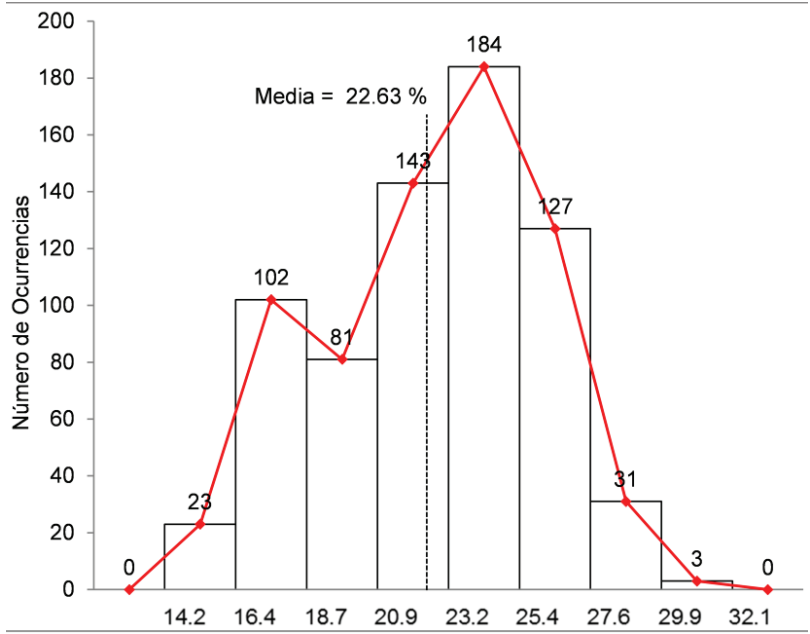

Figura 14. Histograma y polígono de frecuencias para la expansión

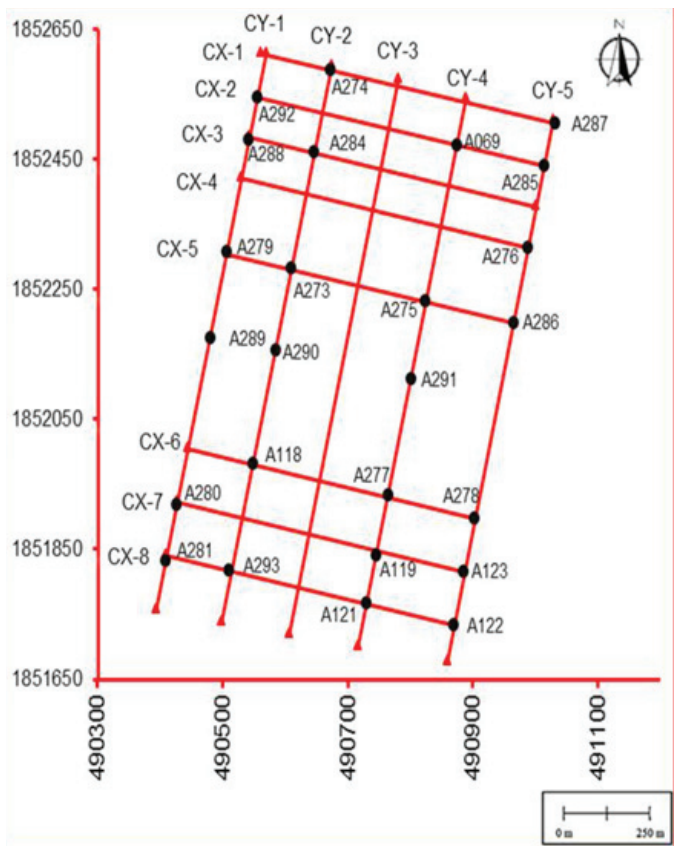

Figura 15. Mapa de ubicación de sondeos y de cortes virtuales 
Tabla 3. Parámetros estadísticos de la expansión

\begin{tabular}{lc}
\hline \multicolumn{2}{c}{ Parámetros estadísticos $\left(\mathrm{E \sigma}_{v^{\prime}} \%\right)$} \\
\hline Media & 1.68 \\
Mediana & 1.51 \\
Moda & 2.06 \\
Desviación estándar & 1.18 \\
Varianza & 1.39 \\
Coeficiente de variación & 0.7 \\
Valor mínimo & 0.11 \\
Valor máximo & 9.86 \\
\hline
\end{tabular}

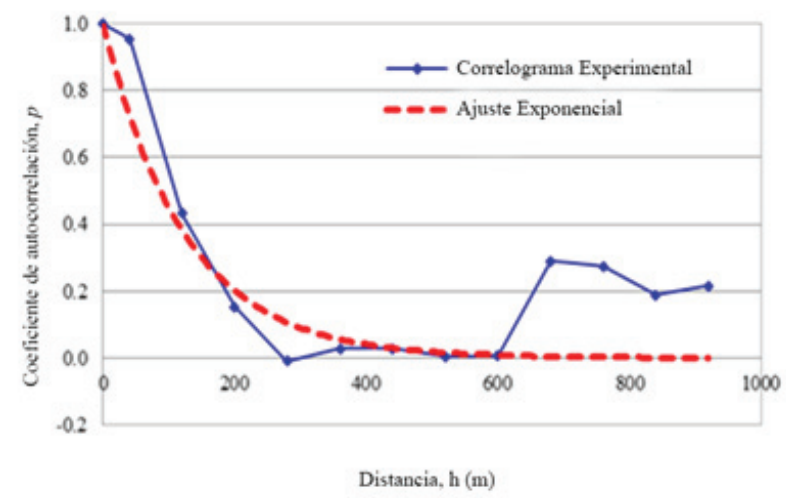

a) Correlograma horizontal, $\delta_{h}=250 \mathrm{~m}$

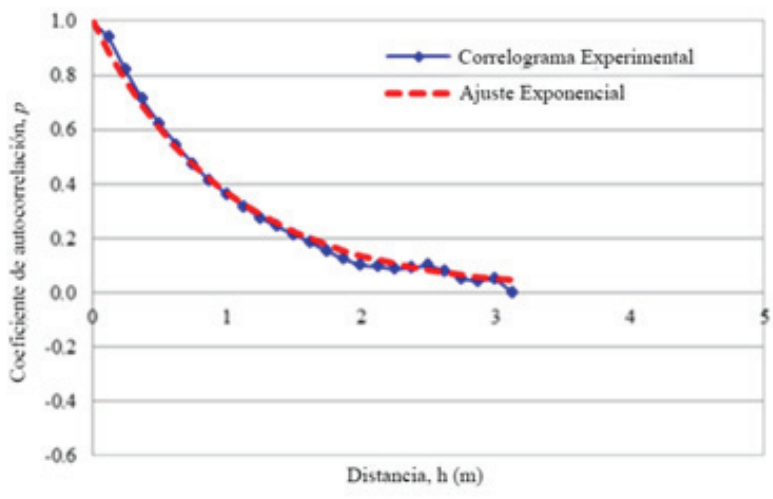

b) Correlograma vertical, $\delta_{v}=2.2 \mathrm{~m}$

Figura 16. Modelos de correlación espacial, contenido de agua

\section{Análisis de la correlación espacial}

Se determinaron las funciones de auto correlación y los modelos de correlación espacial horizontal y vertical. Los correlogramas horizontal y vertical y las distancias de influencia para el contenido de agua y expansión se presentan en la figura 16.

Se determinaron las funciones de auto correlación y los modelos de correlación espacial horizontal y vertical. Los correlogramas horizontal y vertical y las distan- cias de influencia para el contenido de agua y expansión se presentan en la figura 17.

\section{Obtención de cortes virtuales}

Contenido de agua (w, \%)

En la figura 18, se presentan cortes típicos en los que se aprecian la variación espacial del contenido de agua y la desviación estándar de estimación. En el corte CX-2, de 0.25 a $3 \mathrm{~m}$ de profundidad, se observa que el contenido de agua varía de 22 a $25 \%$ y de 3 a $7 \mathrm{~m}$ de profundidad, varía de 24 a $26.5 \%$. De 4 a $7 \mathrm{~m}$ de profundidad (cotas: 490980-491000) el contenido de agua varía de 26.5 a $29 \%$, por la presencia de flujos subterráneos de agua en época de lluvias que resultan visibles aún en época de estiaje. Para el perfil CY-1, En el estrato superior de 0.25 a $3 \mathrm{~m}$, se observa que la variación espacial del contenido de agua es de 21.5 a $24 \%$ y de 3 a $7 \mathrm{~m}$ de profundidad, la variación es de 25.5 a $22 \%$. La zona activa puede definirse a partir de los 4 a $5 \mathrm{~m}$, de profundidad.

\section{Potencial de expansión $\left(E \sigma_{v}, \%\right)$}

En los cortes de la figura 19, se presenta la variación espacial y la desviación estándar de estimación de la expansión. En el corte CX-2, se observan variaciones locales importantes de este parámetro. En los perfiles que registran altos contenidos de agua $w>28 \%$, las expansiones varían de 1.5 a $2.5 \%$ y para contenidos de agua $w<22 \%$, la variación de $E \sigma_{\mathrm{v}}$, es de 5 a $7.5 \%$. En el perfil CY-1, existe continuidad en los perfiles de contenido de agua y en el estrato superior la variación de la expansión unitaria se registran valores en promedio de 5 a $7.5 \%$. Se observan áreas de menor extensión con porcentajes de expansión de 8 a 10\%. En el estrato de 3 a $7 \mathrm{~m}$, en las dos direcciones los perfiles y la variación espacial registran valores de expansión unitaria de 2 a $2.5 \%$, en los perfiles la desviación de estimación $\sigma \rightarrow 0$. Para fines de caracterización geotécnica, estos parámetros de expansión corresponden a un potencial de expansión medio a muy alto.

\section{Mapas de contorno}

Se obtuvieron mapas de contornos de los parámetros de interés en planos ubicados a diferentes profundidades. Se recurrió nuevamente a la geoestadística, ahora en 2D, y a la interpolación mediante el método del kriging. Se definió una malla de puntos en los que se realizó la estimación puntual para cada parámetro geotécnico $(w, \%)$ y $\left(E \sigma_{v} \%\right)$, en dos direcciones. En las figuras 20 (a y b) se presentan los mapas de contornos 


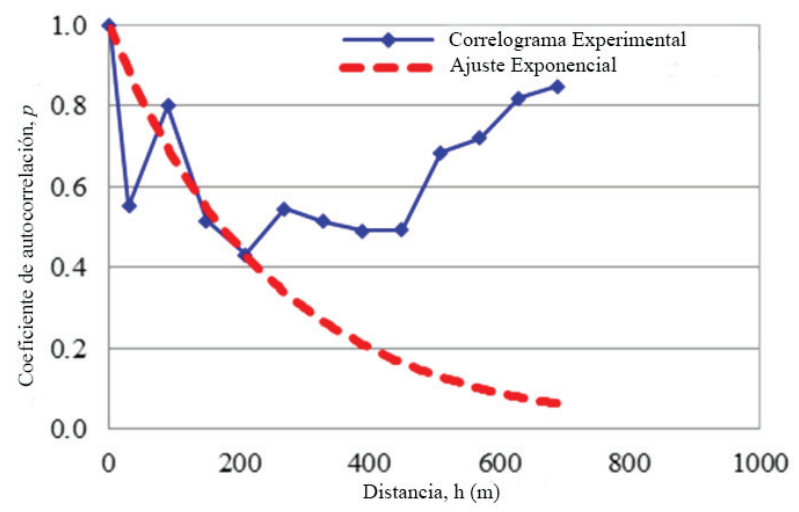

a) Correlograma horizontal, $\delta_{h}=500 \mathrm{~m}$

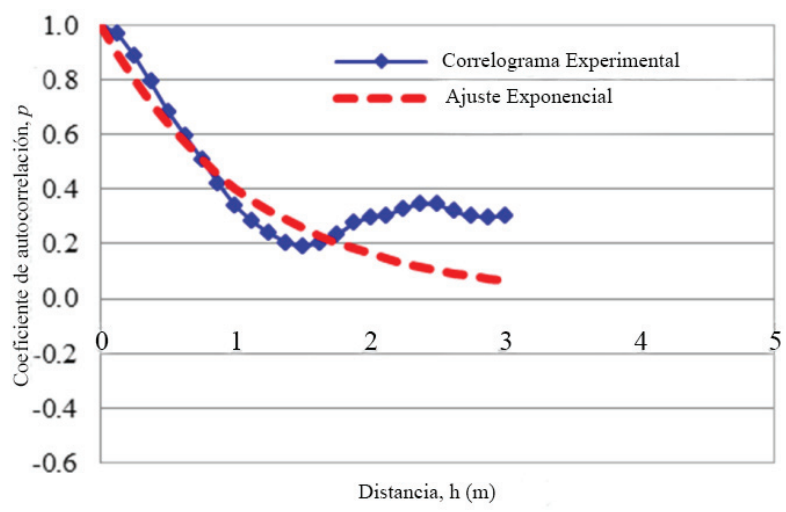

b) Correlograma vertical, $\delta_{v}=2.2 \mathrm{~m}$

Figura 17. Modelos de correlación espacial, expansión

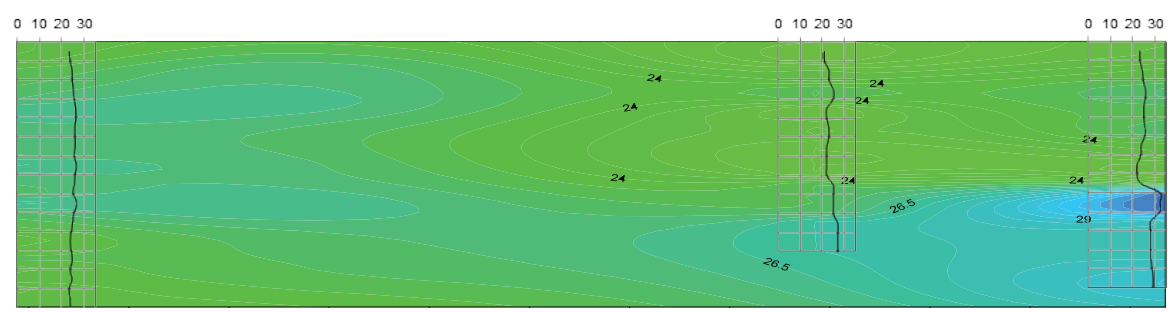

Estimación de corte CX-2

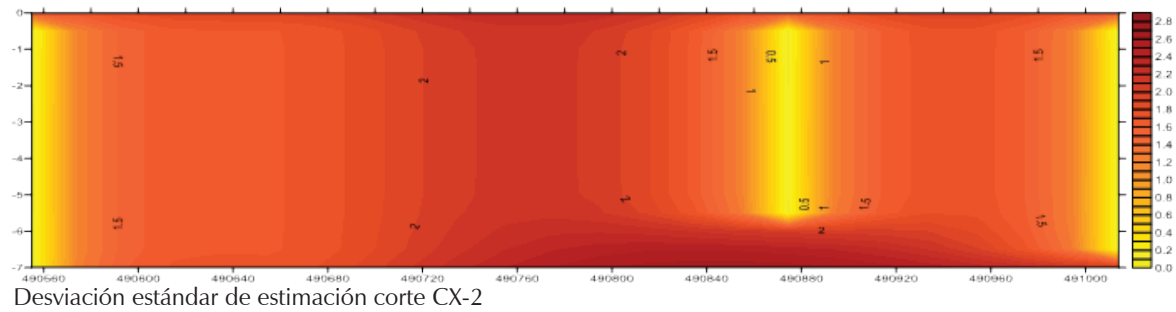

Desviación estándar de estimación corte CX-2

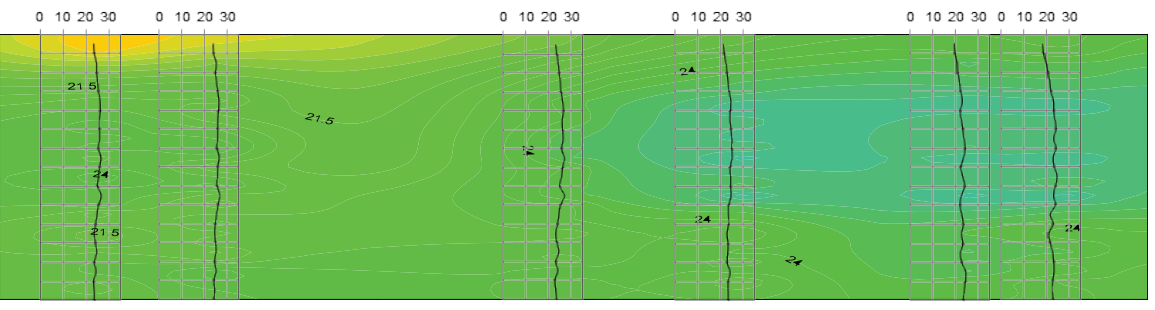

Estimación en el corte CY-1

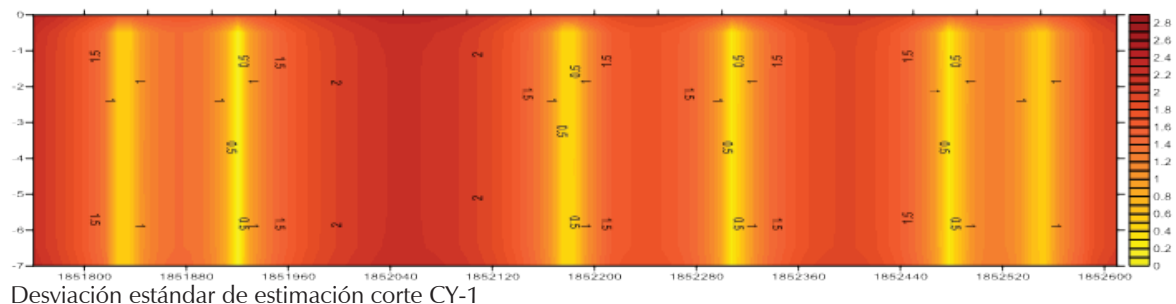

Figura 18. Estimación y desviación estándar de estimación de los cortes CX-2 y CY-1, de ( $w, \%)$ 
para $\mathrm{z}=-0.25,-1,-3$ y $-5 \mathrm{~m}$ de profundidad, para los dos parámetros geotécnicos descritos.

\section{Análisis de riesgos geotécnicos}

La información que se presenta en los incisos anteriores se usó para analizar y clasificar los riesgos geotécnicos y construir mapas de riesgos para el sitio 2. En las tablas 4 y 5 se proponen criterios para la clasificación de los riesgos geotécnicos en suelos expansivos, en función de la diferencia de contenido de agua y potencial de expansión entre épocas de lluvia y estiaje en el mismo punto.
Tabla 4. Criterio para clasificar los riesgos geotécnicos a partir del contenido de agua (Ordóñez, 2013)

\begin{tabular}{cc}
\hline $\begin{array}{c}\text { Parámetros geotécnicos } \\
(\Delta \mathrm{w}, \%)\end{array}$ & $\begin{array}{c}\text { Clasificación de } \\
\text { riesgo geotécnico }\end{array}$ \\
\hline $0-2$ & Bajo \\
$2-5$ & Medio \\
$>5$ & Alto \\
\hline
\end{tabular}

Tabla 5. Criterio para clasificar los riesgos geotécnicos a partir del potencial de expansión (Ordóñez, 2013)

\begin{tabular}{cc}
\hline $\begin{array}{c}\text { Parámetros geotécnicos } \\
(\Delta E \sigma \mathrm{v}, \%)\end{array}$ & $\begin{array}{c}\text { Clasificación de riesgo } \\
\text { geotécnico }\end{array}$ \\
\hline $0-1$ & Bajo \\
$1-2$ & Medio \\
$>2$ & Alto \\
\hline
\end{tabular}

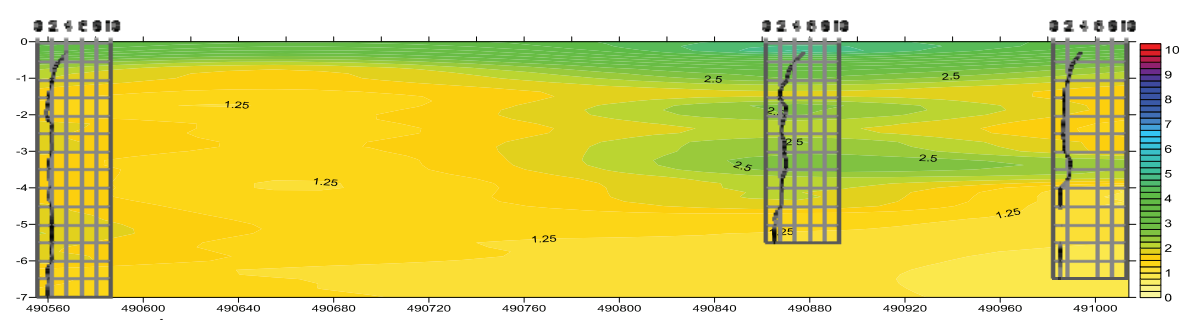

Estimación de corte CX-2

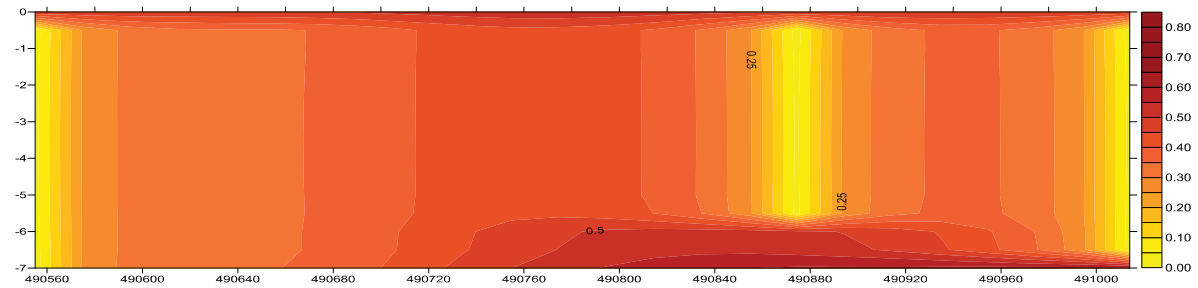

Desviación estándar de estimación corte CX-2

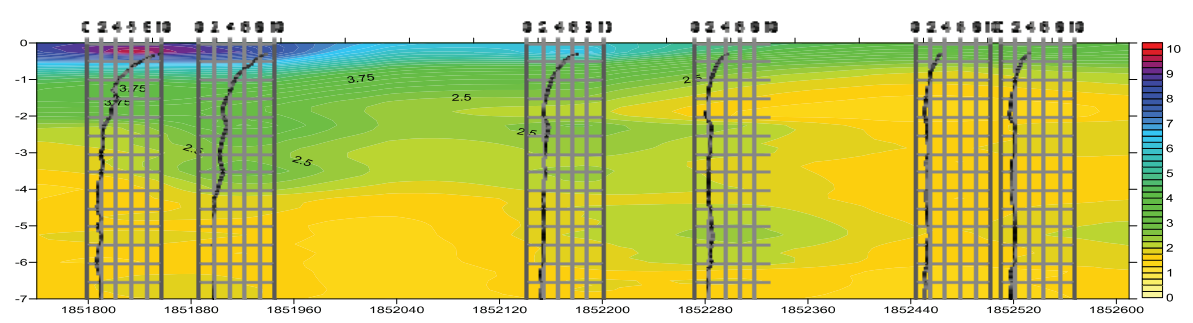

Estimación de corte CY-1

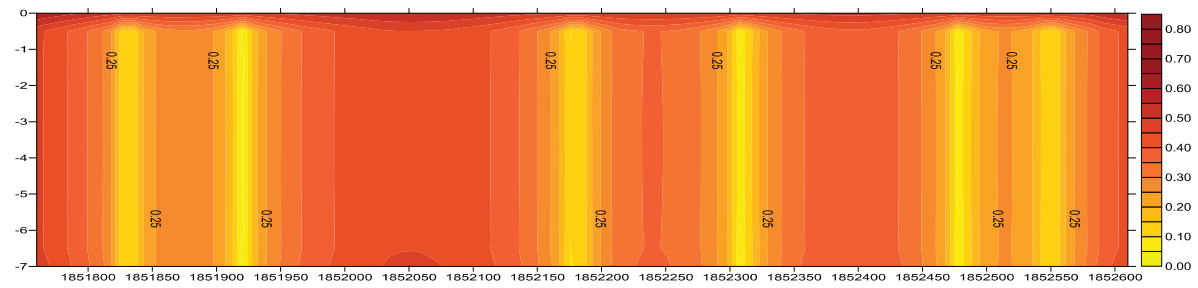

Desviación estándar de estimación corte CY-1
Figura 19. Estimación y desviación estándar de estimación de los cortes CX-2 y $\mathrm{CY}-1$, de $\left(E \sigma_{v}, \%\right)$ 


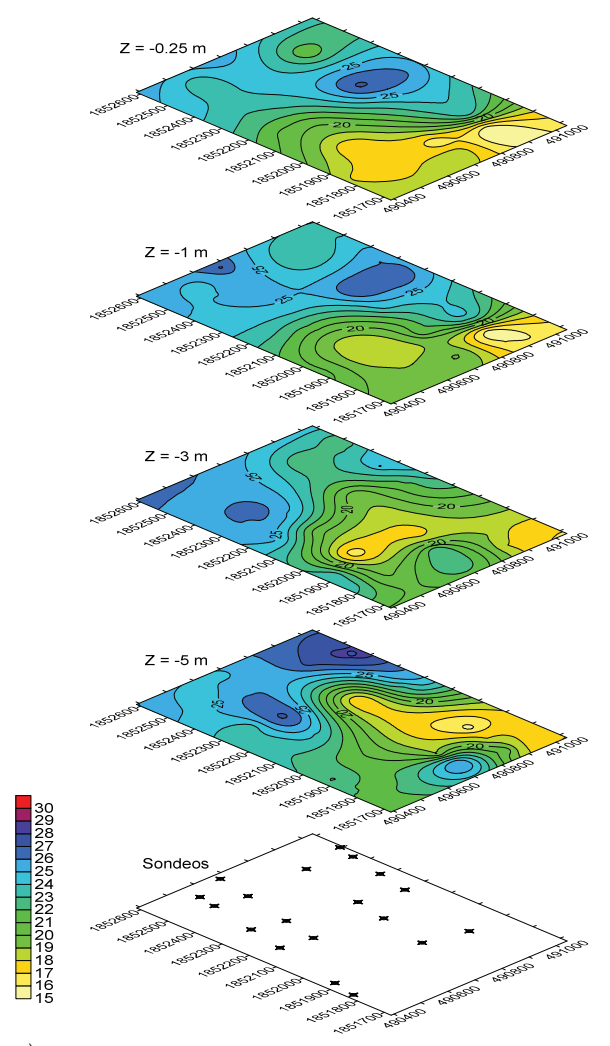

a)

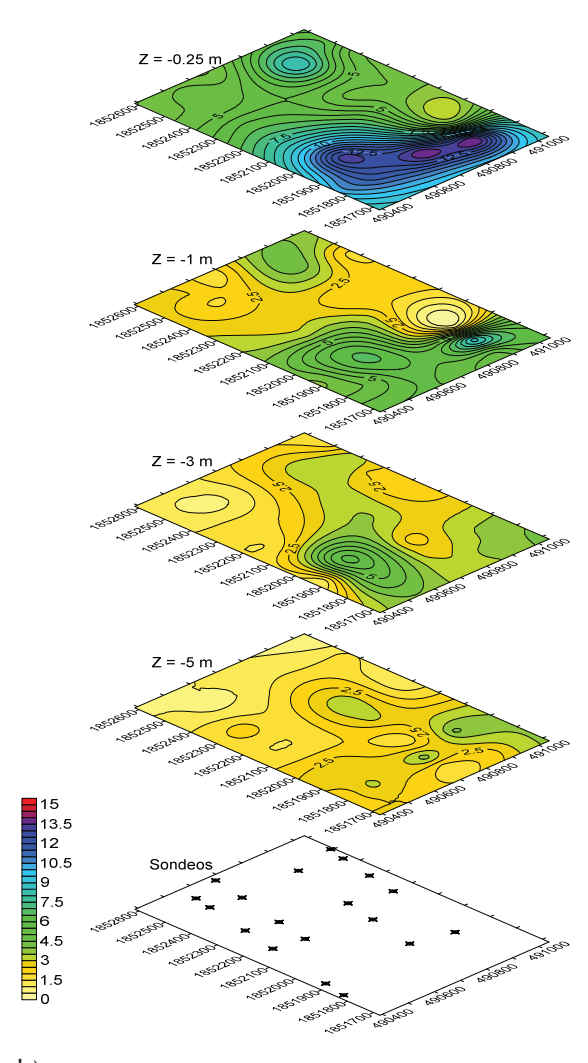

b)

Figura 20. Mapas de contornos para el contenido de agua $(w, \%)$ y la expansión $\left(E \sigma_{v} \%\right)$

En la figura 21a y b, se presentan los mapas de riesgos para $\mathrm{z}=-0.25,-1,-3 \mathrm{y}-5 \mathrm{~m}$ de profundidad, para las condiciones descritas.

\section{Mitigación de riesgos geotécnicos}

Existen diferentes alternativas para la mitigación de riesgos geotécnicos en cimentaciones y pavimentos construidos sobre estratos de arcillas expansivas. Se ha empleado la calhidra y el cemento para la estabilización de suelos expansivos. El problema de esta solución radica en el proceso constructivo que resulta complejo y costoso y requiere un control de calidad riguroso en la elaboración de la mezcla para garantizar la homogeneidad.

Otra alternativa es la estabilización con geomateriales naturales, como son: mezclas de arcilla con arenas y caliche (Ordóñez, 1994) y, recientemente, mezclas de arcillas con granzón (material producto de la trituración de la roca caliza para fabricar agregados). Las experiencias obtenidas al aplicar esta solución se describirán en un próximo trabajo.

\section{Conclusiones}

La investigación se orientó a la caracterización del subsuelo de la ciudad de Tuxtla Gutiérrez y al análisis de riesgos geotécnicos asociados a la presencia de arcillas expansivas.

Se realizó investigación sobre la geología de la región, geología local y geología histórica remontando hasta el Paleoceno. Se contribuye en el presente trabajo al mejor conocimiento geológico de la zona con un mapa de rasgos geoestructurales. Se distinguen: una planicie central delimitada, al sur, por pliegues formados por lutitas $\mathrm{y}$, al norte, por calizas y calizas intercaladas con "lutitas" del cretácico inferior. En el mapa de zonificación geotécnica (Ordóñez, 2008), estos rasgos geológicos permiten definir tres zonas geotécnicas $(\mathrm{A}, \mathrm{B}$ y C). Los suelos expansivos abarcan la mayor parte de la extensión territorial de la zona urbana A y presentan espesores de 0 a 7 y hasta $9 \mathrm{~m}$, de profundidad.

La hidrología del valle se enmarca en la cuenca del río Sabinal conformada por 15 subcuencas y el mismo número de afluentes. Es el dren pluvial más importante de la ciudad y desemboca en el río Grijalva. Los afluentes 


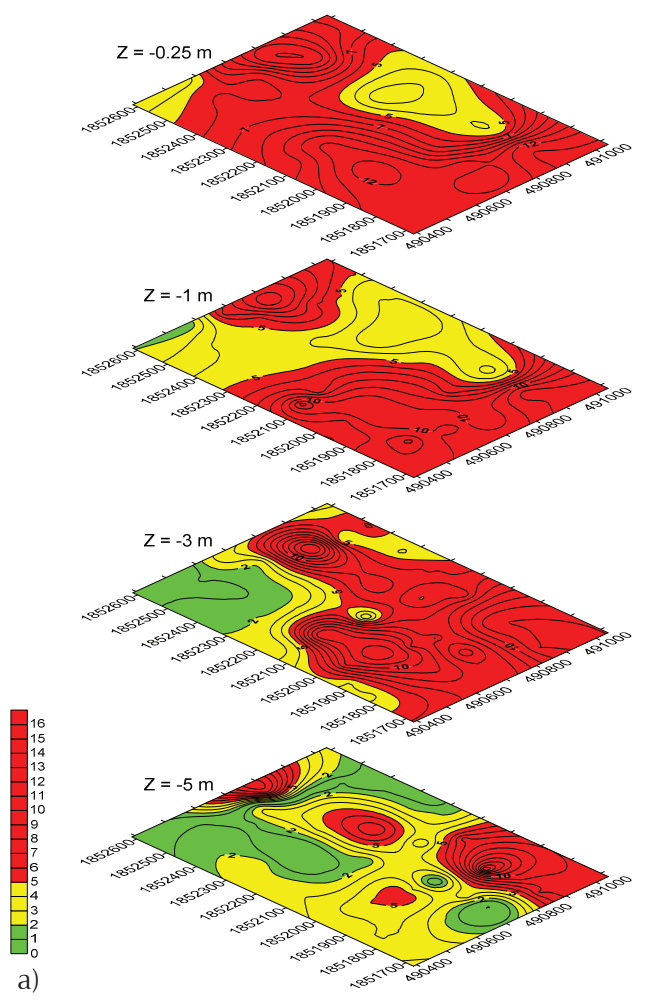

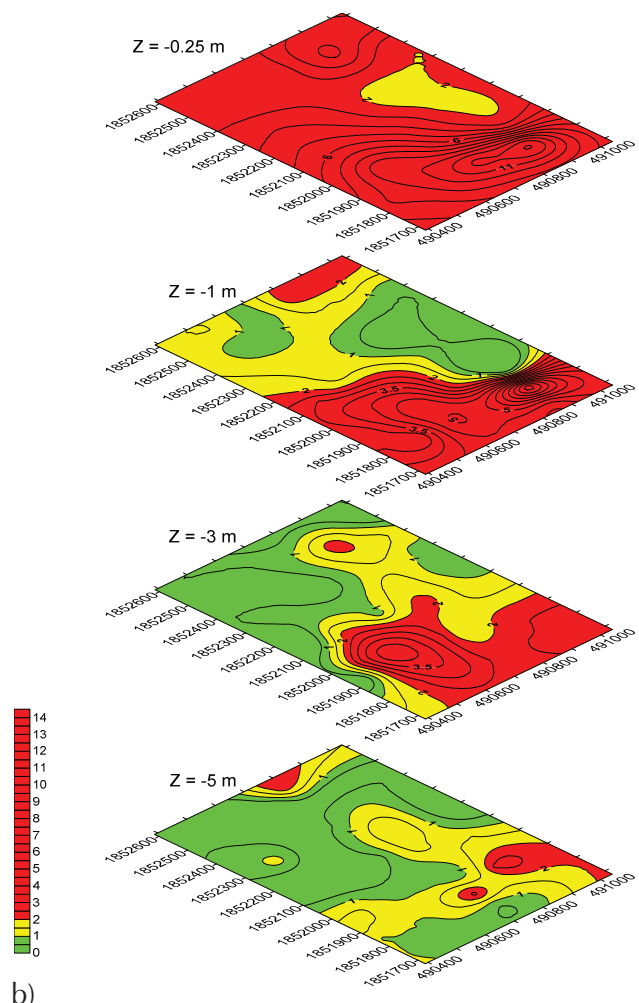

Figura 21. Mapas de riesgos geotécnicos a) para el contenido de agua y b) para el potencial de expansión unitaria del río Sabinal concentran todas las corrientes o flujos de agua del subsuelo de la ciudad que se activan en épocas de lluvias induciendo incrementos de contenido de agua en los estratos de arcillas parcialmente saturadas.

Para ampliar el conocimiento de las características de las arcillas expansivas de la zona A, se elaboró un nuevo programa de investigación experimental de campo y laboratorio. Las propiedades índice de los materiales y la aplicación de los métodos indirectos indican que las arcillas expansivas de la ciudad registran un potencial de expansión: de medio a muy alto. Las muestras inalteradas fueron ensayadas en el consolidómetro en tres modalidades. Los resultados indican que la presión de expansión para: $w_{L}>60 \%$ y $\Delta_{w}<10 \%$, registra valores de $\sigma_{s}>240$ a 420 $\mathrm{kPa}$ y para $\Delta_{w}<5 \%, \sigma_{s}>55 \mathrm{kPa}$. Estos valores de $\sigma_{s^{\prime}}$ indican que en un ciclo de estiaje-lluvias, las arcillas presentan un potencial de expansión de medio a muy alto.

Se realizó el análisis geoestadístico del contenido de agua y expansión. El análisis se ejecutó para las condiciones de estiaje, porque es la condición más crítica para definir el potencial de expansión de los estratos de arcilla. Mediante el método del kriging, se definió la va- riación espacial de las variables geotécnicas contenido de agua $(w, \%)$ y expansión $\left(E \sigma_{v}, \%\right)$. Los mapas de contornos y de riesgos geotécnicos obtenidos indican que para las profundidades de 0.25 a $3 \mathrm{~m}$ predomina el riesgo de medio a alto y en el mapa de $5 \mathrm{~m}$ predomina el riesgo bajo a medio.

\section{Agradecimientos}

Al Instituto de Ingeniería de la UNAM y a la Universidad Autónoma de Chiapas por las facilidades otorgadas para la estancia en el Instituto de Ingeniería. A la empresa Geortec, por su apoyo en la parte experimental de este trabajo.

\section{Anexo 1}

Sondeos realizados en época de lluvias para los sitios 1 y 2, para determinar las propiedades índice: sitio 1 (datos: 26-48) sitio 2 (datos: 1-25). Con estos parámetros se determina el potencial de expansión de las arcillas de la ciudad de Tuxtla Gutiérrez. 


\begin{tabular}{|c|c|c|c|c|c|c|c|c|c|c|c|c|c|c|c|c|c|}
\hline \multicolumn{2}{|c|}{$\begin{array}{l}\text { Espesor de } \\
\text { estrato }\end{array}$} & \multicolumn{8}{|c|}{$0.25-1.5 \mathrm{~m}$} & \multicolumn{8}{|c|}{$1.75-4 \mathrm{~m}$} \\
\hline Datos & $\begin{array}{c}\text { Clave de } \\
\text { sondeo }\end{array}$ & $\begin{array}{l}\mathrm{W}_{\mathrm{L}} \\
(\%)\end{array}$ & $\begin{array}{l}\mathrm{W}_{\mathrm{p}} \\
(\%)\end{array}$ & $\begin{array}{l}\mathrm{W}_{\mathrm{S}} \\
(\%)\end{array}$ & $\begin{array}{c}\mathrm{S}_{\mathrm{L}} \\
(\%)\end{array}$ & $\begin{array}{l}(\%) \\
2 \mu\end{array}$ & $\begin{array}{c}\text { IS } \\
(\%)\end{array}$ & $\begin{array}{c}\text { PI } \\
(\%)\end{array}$ & A & $\begin{array}{l}W_{L} \\
(\%)\end{array}$ & $\begin{array}{l}\mathrm{W}_{\mathrm{p}} \\
(\%)\end{array}$ & $\begin{array}{l}\mathrm{W}_{\mathrm{S}} \\
(\%)\end{array}$ & $\begin{array}{c}\mathrm{S}_{\mathrm{L}} \\
(\%)\end{array}$ & $\begin{array}{l}(\%) \\
2 \mu\end{array}$ & $\begin{array}{l}\text { IS } \\
(\%)\end{array}$ & $\begin{array}{c}\text { PI } \\
(\%)\end{array}$ & A \\
\hline 1 & A 240 & 70 & 21 & 10.75 & 19 & 53 & 59.3 & 49 & 1.02 & 68 & 23 & 11.42 & 19 & 65 & 57 & 46 & 0.8 \\
\hline 2 & A241 & 60 & 20 & 13.75 & 16 & 62 & 46.0 & 40 & 0.70 & 68 & 23 & 11.50 & 18 & 62 & 56 & 45 & 0.8 \\
\hline 3 & A242 & 63 & 23 & 12.50 & 15 & 51 & 50.2 & 40 & 0.87 & 70 & 22 & 10.75 & 18 & 51 & 59 & 48 & 1.1 \\
\hline 4 & A243 & 66 & 23 & 11.75 & 18 & 51 & 54.0 & 43 & 0.94 & 70 & 24 & 11.05 & 18 & 51 & 59 & 46 & 1.0 \\
\hline 5 & A244 & 63 & 22 & 12.15 & 16 & 62 & 51.1 & 41 & 0.73 & 71 & 24 & 10.30 & 19 & 62 & 61 & 47 & 0.8 \\
\hline 6 & A245 & 60 & 19 & 13.25 & 17 & 65 & 46.9 & 41 & 0.69 & 60 & 19 & 13.25 & 17 & 65 & 47 & 41 & 0.7 \\
\hline 7 & A246 & 52 & 16 & 16.15 & 11 & 60 & 35.4 & 35 & 0.64 & 62 & 19 & 12.50 & 16 & 60 & 50 & 43 & 0.8 \\
\hline 8 & A247 & 63 & 24 & 12.50 & 16 & 62 & 50.3 & 39 & 0.68 & 64 & 23 & 12.15 & 16 & 62 & 52 & 41 & 0.7 \\
\hline 9 & A248 & 60 & 20 & 13.75 & 16 & 60 & 46.1 & 40 & 0.73 & 60 & 20 & 13.75 & 16 & 60 & 46 & 40 & 0.7 \\
\hline 10 & A249 & 59 & 23 & 14.50 & 14 & 51 & 44.4 & 36 & 0.79 & 62 & 21 & 13.25 & 16 & 51 & 48 & 40 & 0.9 \\
\hline 11 & A270 & 64 & 19 & 11.75 & 15 & 60 & 52.3 & 45 & 0.82 & 66 & 21 & 11.50 & 17 & 60 & 55 & 46 & 0.8 \\
\hline 12 & $\mathrm{~A} 271$ & 62 & 20 & 12.50 & 15 & 62 & 49.9 & 43 & 0.75 & 65 & 20 & 11.75 & 16 & 62 & 53 & 45 & 0.8 \\
\hline 13 & A250 & 65 & 16 & 12.15 & 19 & 53 & 52.5 & 49 & 1.01 & 72 & 18 & 10.30 & 21 & 53 & 62 & 53 & 1.1 \\
\hline 14 & A251 & 55 & 18 & 15.30 & 16 & 62 & 39.8 & 37 & 0.65 & 72 & 18 & 10.30 & 21 & 53 & 62 & 53 & 1.1 \\
\hline 15 & A252 & 63 & 24 & 12.15 & 15 & 53 & 51.0 & 39 & 0.81 & 62 & 23 & 12.50 & 14 & 53 & 50 & 39 & 0.8 \\
\hline 16 & A294 & 57 & 19 & 15 & 16 & 60 & 41.7 & 38 & 0.69 & 52 & 20 & 16.15 & 16 & 60 & 36 & 32 & 0.6 \\
\hline 17 & A295 & 59 & 20 & 13.8 & 16 & 60 & 45.4 & 39 & 0.71 & 51 & 15 & 16.5 & 14 & 60 & 34 & 36 & 0.6 \\
\hline 18 & A296 & 43 & 17 & 22 & 7 & 51 & 21.0 & 26 & 0.58 & 59 & 19 & 13.75 & 16 & 51 & 45 & 40 & 0.9 \\
\hline 19 & A297 & 51 & 19 & 16.2 & 14 & 58 & 35.1 & 33 & 0.62 & 53 & 20 & 15.75 & 14 & 58 & 37 & 33 & 0.6 \\
\hline 20 & A298 & 45 & 16 & 21.5 & 11 & 52 & 23.4 & 28 & 0.61 & 61 & 19 & 13.25 & 13 & 52 & 48 & 42 & 0.9 \\
\hline 21 & A299 & 51 & 20 & 16.5 & 11 & 58 & 34.1 & 30 & 0.57 & 62 & 21 & 13.25 & 16 & 58 & 48 & 41 & 0.8 \\
\hline 22 & A230 & 60 & 18 & 13.8 & 15 & 62 & 45.8 & 41 & 0.72 & 69 & 19 & 11.5 & 17 & 62 & 57 & 49 & 0.9 \\
\hline 23 & A231 & 61 & 22 & 13.3 & 16 & 62 & 47.7 & 39 & 0.68 & 57 & 19 & 14.75 & 15 & 62 & 42 & 38 & 0.7 \\
\hline 24 & A232 & 60 & 22 & 13.3 & 16 & 60 & 46.7 & 38 & 0.70 & 57 & 22 & 14.75 & 14 & 60 & 42 & 35 & 0.6 \\
\hline 25 & A233 & 57 & 17 & 14.8 & 15 & 60 & 42.7 & 40 & 0.73 & 62 & 19 & 12.5 & 17 & 60 & 50 & 43 & 0.8 \\
\hline 26 & A101 & 69 & 23 & 9.85 & 17 & 58 & 59.3 & 46 & 0.87 & 63 & 19 & 9.88 & 18 & 65 & 53 & 44 & 0.7 \\
\hline 27 & A102 & 64 & 20 & 9.74 & 19 & 65 & 54.3 & 45 & 0.75 & 60 & 19 & 9.89 & 18 & 65 & 50 & 41 & 0.7 \\
\hline 28 & A103 & 64 & 19 & 10.64 & 18 & 65 & 53.4 & 45 & 0.75 & 64 & 18 & 10.4 & 19 & 65 & 54 & 46 & 0.8 \\
\hline 29 & A104 & 66 & 19 & 10.64 & 18 & 65 & 55.4 & 47 & 0.78 & 60 & 18 & 10.4 & 18 & 65 & 50 & 42 & 0.7 \\
\hline 30 & A105 & 66 & 19 & 10.85 & 18 & 63 & 55.2 & 47 & 0.81 & 64 & 17 & 10.4 & 20 & 63 & 54 & 47 & 0.8 \\
\hline 31 & A106 & 68 & 21 & 10.05 & 18 & 61 & 58.0 & 47 & 0.84 & 65 & 20 & 11 & 18 & 61 & 54 & 45 & 0.8 \\
\hline 32 & A107 & 64 & 20 & 10.75 & 18 & 59 & 53.3 & 44 & 0.81 & 69 & 22 & 11 & 19 & 59 & 58 & 47 & 0.9 \\
\hline 33 & A108 & 68 & 20 & 10.50 & 19 & 60 & 57.5 & 48 & 0.87 & 59 & 16 & 11.5 & 17 & 60 & 47 & 43 & 0.8 \\
\hline 34 & A019 & 61 & 18 & 10.75 & 17 & 63 & 50.3 & 43 & 0.74 & 64 & 18 & 10.40 & 19 & 63 & 54 & 46 & 0.8 \\
\hline 35 & A120 & 59 & 15 & 11.90 & 17 & 63 & 46.8 & 44 & 0.75 & 62 & 17 & 11.20 & 18 & 63 & 50 & 45 & 0.8 \\
\hline 36 & A121 & 62 & 17 & 10.75 & 18 & 60 & 51.1 & 45 & 0.81 & 57 & 15 & 12.00 & 17 & 60 & 45 & 42 & 0.8 \\
\hline 37 & A122 & 60 & 15 & 11.3 & 20 & 58 & 48.5 & 45 & 0.84 & 58 & 16 & 11.50 & 22 & 58 & 47 & 42 & 0.8 \\
\hline 38 & A123 & 63 & 20 & 10.55 & 20 & 60 & 52.2 & 43 & 0.77 & 62 & 20 & 10.50 & 21 & 60 & 51 & 42 & 0.8 \\
\hline 39 & A124 & 62 & 20 & 10.75 & 19 & 60 & 51.0 & 42 & 0.76 & 64 & 19 & 10.25 & 23 & 60 & 54 & 45 & 0.8 \\
\hline 40 & A125 & 60 & 16 & 10.30 & 21 & 63 & 49.5 & 44 & 0.76 & 50 & 12 & 10.30 & 21 & 63 & 40 & 38 & 0.7 \\
\hline 41 & A126 & 57 & 13 & 11.50 & 21 & 60 & 45.3 & 43 & 0.79 & 60 & 15 & 10.80 & 23 & 60 & 49 & 45 & 0.8 \\
\hline 42 & A127 & 62 & 16 & 10.00 & 21 & 60 & 52.0 & 46 & 0.83 & 62 & 17 & 11.05 & 18 & 60 & 51 & 45 & 0.8 \\
\hline 43 & A128 & 62 & 18 & 10.00 & 18 & 60 & 52.0 & 44 & 0.80 & 67 & 19 & 11.05 & 18 & 60 & 56 & 49 & 0.9 \\
\hline 44 & A129 & 69 & 16 & 10.00 & 18 & 61 & 58.8 & 53 & 0.94 & 67 & 19 & 10.00 & 18 & 61 & 57 & 48 & 0.9 \\
\hline 45 & A130 & 68 & 22 & 10.55 & 17 & 61 & 57.0 & 46 & 0.82 & 64 & 23 & 11.05 & 17 & 61 & 53 & 41 & 0.7 \\
\hline 46 & A131 & 70 & 22 & 10.00 & 46 & 60 & 59.8 & 48 & 0.86 & 68 & 22 & 11.05 & 46 & 60 & 57 & 46 & 0.8 \\
\hline 47 & A132 & 58 & 16 & 12.75 & 16 & 60 & 45.4 & 42 & 0.77 & 59 & 17 & 12.75 & 16 & 60 & 46 & 42 & 0.8 \\
\hline 48 & A133 & 59 & 16 & 12.75 & 17 & 60 & 46.0 & 43 & 0.78 & 59 & 17 & 12.75 & 17 & 60 & 46 & 42 & 0.8 \\
\hline
\end{tabular}

\section{Referencias}

ASTM. Special procedures for Testing Soil and Rocks for Engineering Purposes, American Society for Testing and Materials, Special Technical Publication, 483, 1971.

Auvinet G. Incertidumbre en Geotecnia, XVI Conferencia Nabor Carrillo, Sociedad Mexicana de Mecánica de Suelos, México, DF, 2000.

Bishop A.W. y Blight G.E. Some aspect of effective stress in saturated and unsaturated Soil. Geotechnique, volumen 13, número 3, 1963:177-1197.

Bishop A.W. y Blight G.E. The principle of Effective Stress, lecture delivered in Oslo. Norway. published in Teknisk Ukebland, volumen 106 (número 39), 1959: 859-863.

Casagrande A. Classification and identificacion of soils-American Society of Civil Engineers-Trans, volumen 113, 1948.
Dumbleton M.S. The classification and description of soil for engineering purpose, british system RRL, Report LR 182, United Kingdom, 1968.

Dakshanamurthy V. y Raman V. A simple method of identifying on expansive soil. Soil and Foundation, Japanese Society of Soil Mechanics and Foundation Engineering, volumen 13 (número 1), 1973: 97-104.

De la Rosa Z.J.L.y Aldemar E.S. Geología del Estado de Chiapas, México, 1989.

Domínguez R. et al. Análisis del sistema principal de drenaje y control de avenidas de la ciudad de Tuxtla Gutiérrez, Ed. (CNA), Chiapas, México, 1997.

Fredlund D.G. y Rahardjo H. Soil Mechanics for Unsaturated Soils, Canadá, John Wiley \& Sons, Inc., 1993.

Fredlund D.G. y Morgenstern N.R. The shear strength of unsaturated soils. Canadian Geotechnical Journal, volumen 15 (número 3), 1977. 
Información Geográfica y Estadística. INEGI, Ed. INEGI, México, DF, 2009.

Lambe T. W. y Whitman R.V. Soil mechanics, Wiley, Nueva York, 1969.

López E.G. Estimación de tormentas y avenidas para el diseño de las obras de protección del río Sabinal, (tesis de maestría), UNAM, México, DF, 2006

Matheron G. Traite de Géostatistique Appliquée, Tome I, Mémoires Bureau de Recherches Géologiques et Minières, Núm. 24, Paris. Francia, Editions Bureau de Recherches Géologiques et Minières, 1962.

Merwe D.H. Current theory and practice for building on expansive clays, proceedings of the 6th Regional Conference for Africa on soil mechanics and foundation engineering, Durban, volumen 2, 1964 y 1975, pp.166-167.

Merwe D.H. Current Theory and practice for building on expansive clays, proceedings of the 6th Regional Conference for Africa on Soil Mechanics and Foundation Engineering, Durban, volumen 2, 1975, pp.166-167.

Ordóñez J. Estabilización de las arcillas expansivas con caliche y arena en la Ciudad de Tuxtla Gutiérrez, (tesis de maestría), IPN, México, 1994.

Ordóñez R.J. Zonificación geotécnica de la Ciudad de Tuxtla Gutiérrez, Facultad de Ingeniería, UNACH, Chiapas, México, 2008.

Ordóñez J. Criterio para clasificar los tipos de riesgos geotécnicos para suelos expansivos. Instituto de Ingeniería, UNAM, México, DF, 2013.

Seed H., Woodward R.J., Lundgren R. Prediction of swelling potential for compacted clays. Proceedings of the ASCE. Journal of the Soil Mechanics and Foundation Division, volumen 88, (SM3), 1962: 53-87.

Terzaghi K. The shear resistance of saturated soils, on: Proc. 1st, International Conference Soils Mechanics, Foundation Engineering, Cambridge MA, volumen 1, 1936, pp. 54-56.

\section{Bibliografía}

Altmeyer W.T. Discussion of engineering properties of expansive clays. Proceedings ASCE, volumen 81 (número 658), marzo de 1995.
Chen F.H. Foundation expansive soils. Elsevier Scientific Publishing Company, USA, 1988.

Deutsch C. y Journel A. Geostatistical Software Library, GSLIB, Oxford University Press, Nueva York, USA, 1992.

Figueroa J. Sismicidad en Chiapas, Informe del Instituto de Ingeniería, UNAM, México, 1974.

Holtz W.G. Expansive Clays, Properties and problems. Quarterly of the Colorado School of Mines, volumen 54 (número 4), 1959: $89-125$.

Nelson J. y Miller D. Expansive soil problems and practice in foundation and pavement engineering, John Wiley \& Sons Inc, Nueva York, 1992.

Ordóñez J. Las arcillas expansivas de la Ciudad de Tuxtla Gutiérrez, Memorias de la XXI Reunión Nacional de Mecánica de Suelos, Santiago de Querétaro, México, 2002.

Pousada P.E. Deformabilidad de las arcillas expansivas bajo succión controlada, Madrid, España, Ed. Ministerio de Obras Públicas y Transportes de España, Gabinete de Formación y Documentación, 1984.

Zeevaert L. Foundation engineering for difficult subsoil conditions, 2a ed., Nueva York, Van Nostrand-Reynhold Company, 1982.

\footnotetext{
Este artículo se cita:

Citación estilo Chicago

Ordóñez-Ruiz, Jorge, Gabriel Auvinet-Guichard, Moisés Juárez-Camarena. Caracterizacion del subsuelo y análisis de riesgos geotécnicos asociados a las arcillas expansivas de la ciudad de Tuxtla Gutiérrez. Ingeniería Investigación y Tecnología, XVI, 03 (2015): 453-470.

\section{Citación estilo ISO 690}

Ordóñez-Ruiz J., Auvinet-Guichard G., Juárez-Camarena M. Caracterización del subsuelo y análisis de riesgos geotécnicos asociados a las arcillas expansivas de la ciudad de Tuxtla Gutiérrez. Ingeniería Investigación y Tecnología, volumen XVI (número 3), julio-septiembre 2015: 453-470.
}

\section{Semblanzas de los autores}

Jorge Ordóñez-Ruiz. Ingeniero civil por la Universidad Autónoma de Chiapas (Un.A.Ch.), obtuvo la maestría en ciencias (mecánica de suelos) por la ESIA-IPN. Candidato a doctor en ingeniería (mecánica de suelos) en el Instituto de Ingeniería, UNAM. Actualmente es profesor e investigador de la Un.A.Ch.

Gabriel Auvinet-Guichard. Doctor por la Facultad de Ingeniería de la UNAM. Investigador del Instituto de Ingeniería y profesor en el programa de maestría y doctorado en ingeniería de la UNAM. Ha sido presidente de la Sociedad Mexicana de Mecánica de Suelos y vice-presidente por Norte América de la Sociedad Internacional de Mecánica de Suelos e Ingeniería Geotécnica. Actualmente dirige el Laboratorio de Geoinformática del Instituto de Ingeniería de la UNAM.

Moisés Juárez Camarena. Ingeniería civil y maestría en ciencias (mecánica de suelos) por la Escuela Superior de Ingeniería y Arquitectura (ESIA), IPN. Candidato a doctor en ingeniería (mecánica de suelos) en el Instituto en Ingeniería, UNAM y miembro de la Sociedad Internacional de Mecánica de Suelos e Ingeniería Geotécnica, donde participa en el "Technical Committee on Geotechnical Infrastructure for Megacities and New Capitals". 